\title{
Impacto da invasão e do manejo do capim-gordura (Melinis minutiflora) sobre a riqueza e biomassa da flora nativa do Cerrado sentido restrito
}

\author{
CARLOS ROMERO MARTINS ${ }^{1,6}$, JOHN DU VALL HAY², BRUNO MACHADO TELES WALTER ${ }^{3}$, \\ CAROLYN ELINORE BARNES PROENÇA ${ }^{4}$ e LUCIO JOSÉ VIVALDI ${ }^{5}$
}

(recebido: 19 de agosto de 2010; aceito: 20 de janeiro de 2011)

\begin{abstract}
Impact of invasion and management of molasses grass (Melinis minutiflora) on the native vegetation of the Brazilian Savanna). In Brazil, several grass species are cited as invaders of protected areas. However, little is known about the impacts due to establishment and colonization of these species in protected areas in Brazil. Among the exotic grasses introduced into the Cerrado the African species Melinis minutiflora P. Beauv., molasses grass, deserves special mention. The objective of this study was to evaluate the impact of this grass on the biomass and species richness of the native community in an area of invaded Cerrado as well as to study the dynamics of the vegetation of the ground layer after different management treatments for control of molasses grass. The results showed that in the experimental area, where molasses grass composed $62 \%$ of the total biomass of the ground layer, the number of native species was high. In the areas where molasses grass had a high degree of coverage (>98\%) its biomass was approximately two times higher than values cited in other studies in the Cerrado. Between three and four years after using fire as a management tool for control of molasses grass its biomass returned to values similar to those observed prior to this treatment. Contrarily, with an integrated management treatment (May or September) a reduction of $99.9 \%$ in the presence of molasses grass was observed along with a recovery of native vegetation, making this a promising strategy for recuperation of areas in the Cerrado that were invaded by molasses grass.
\end{abstract}

Key words - biological invasions, conservation, restoration

RESUMO - (Impacto da invasão e do manejo do capim-gordura (Melinis minutiflora) sobre a riqueza e biomassa da flora nativa do Cerrado sentido restrito). No Brasil, várias espécies de gramíneas são citadas como invasoras em Unidades de Conservação. Contudo, ainda se conhece muito pouco sobre o impacto do estabelecimento e da colonização dessas espécies nas áreas protegidas. Entre as gramíneas exóticas introduzidas no bioma Cerrado merece destaque a espécie africana Melinis minutiflora P. Beauv., o capim-gordura. O presente estudo objetivou avaliar o impacto desta invasora na biomassa e na riqueza da comunidade nativa em uma área de Cerrado Ralo invadido, como também estudar a dinâmica da vegetação do estrato rasteiro submetida à aplicação de diferentes técnicas de manejo para o controle do capim-gordura. Os resultados mostraram que, na área experimental, onde o capimgordura representa cerca $62 \%$ da biomassa total do estrato rasteiro, o número de espécies nativas encontradas foi alto. Nas áreas onde o capim-gordura apresentou alto índice de colonização (> 98\%), sua biomassa alcançou cerca de duas vezes a biomassa do estrato rasteiro registrada para o Cerrado. A realização de uma queimada não foi suficiente para controlar o capim-gordura, porque após três anos a sua biomassa se aproximou aos valores encontrados inicialmente. Por outro lado, no tratamento manejo integrado (maio ou setembro) a redução de mais de $99,9 \%$ na sua presença favoreceu a expansão da vegetação nativa, configurando-se, assim, uma estratégia promissora para a recuperação ambiental das áreas invadidas pelo capim-gordura no Cerrado.

Palavras-chave - conservação, invasão biológica, recuperação ambiental

\section{Introdução}

O processo de invasão biológica tem sido responsável por mudanças significativas na estrutura e

1. Instituto Brasileiro do Meio Ambiente e dos Recursos Naturais Renováveis, SCEN Trecho 2, Caixa Postal 09870, Asa Norte, 70.818-900 Brasília, DF, Brasil.

2. Universidade de Brasília, Instituto de Ciências Biológicas, Departamento de Ecologia, Asa Norte, 70.910-900 Brasília, DF, Brasil.

3. Embrapa Recursos Genéticos e Biotecnologia, Herbário CEN, Caixa Postal 02372, 70.770-900 Brasília, DF, Brasil.

4. Universidade de Brasília, Instituto de Ciências Biológicas, Departamento de Botânica, Asa Norte, 70.910-900 Brasília, DF, Brasil.

5. Universidade de Brasília, Instituto de Ciências Exatas, Departamento de Estatística, Asa Norte, 70.910-900 Brasília, DF, Brasil.

6. Autor para correspondência: carlos.martins@ibama.gov.br na composição da vegetação nos ambientes naturais em diferentes partes do mundo (San-José \& Fariñas 1991, D'Antonio \& Vitousek 1992, McNeely et al. 2001). No Brasil, várias espécies são citadas como invasoras em Unidades de Conservação, embora pouco se conheça sobre o impacto decorrente do estabelecimento e da colonização dessas espécies nas áreas protegidas (Pivello et al. 1999a, Martins et al. 2007).

A gramínea africana Melinis minutiflora P. Beauv. (capim-gordura) é uma invasora extremamente agressiva, que compete com sucesso com a flora nativa e, em particular, com a flora do Cerrado sentido amplo (lato sensu). Além de se estabelecer em áreas antropizadas, também é capaz de invadir áreas naturais e, em poucos anos, descaracterizar a fitofisionomia original (Filgueiras 
1990, Martins et al. 2004). No bioma Cerrado é considerada uma das principais espécies invasoras e, devido ao seu potencial invasor, tornou-se um grande problema nas Unidades de Conservação (Pivello et al. 1999a, 1999b, Martins et al. 2007).

No Distrito Federal, com uma área de $5.814 \mathrm{~km}^{2}$, os focos mais importantes de capim-gordura em áreas protegidas estão localizados no Parque Nacional de Brasília (PNB), na Estação Ecológica de Águas Emendadas, Fazenda Água Limpa (FAL/Universidade de Brasília), Reserva Biológica da Contagem (R) e Reserva Biológica do Instituto Brasileiro de Geografia e Estatística (IBGE). Porém, poucas são as informações sobre o percentual dessas áreas que estão ocupadas por essa gramínea (Martins et al. 2004).

Conforme informações oriundas de Miditieri (1983), Martins et al. (2004) e Martins (2006), o capim-gordura é uma espécie perene, herbácea, apomítica e possui metabolismo $\mathrm{C}_{4}$. Apresenta altura média entre 0,40 e $0,60 \mathrm{~m}$, podendo atingir 1,6 m ou mais. Os colmos ascendentes são provenientes de uma base emaranhada e pouco ramificada. Emite raízes nos entrenós inferiores e tende, comumente, a dobrar-se. Os pelos glandulares da folhagem exudam óleo com cheiro característico. Sua inflorescência é do tipo panícula terminal (estreita) e mede entre 10 a $30 \mathrm{~cm}$ de comprimento. Dependendo da variedade cultivada, sua floração ocorre entre 166 a 202 dias após a semeadura. As sementes são muito pequenas $(1,5$ a 2,5 mm), leves $(0,00010$ a 0,00013 g) e são providas de aristas, o que facilita a sua disseminação ectozoocórica e anemocórica. Produz grande quantidade de sementes (200-280 $\left.\mathrm{kg} \mathrm{ha}^{-1}\right)$ e, em um quilograma, são encontradas entre 13 a 15 milhões de sementes. As sementes apresentam alto poder de germinação e alta dormência de longa duração.

Entre as gramíneas exóticas presentes no PNB a que mais se destaca é o capim-gordura, devido à grande área que ocupa. Atualmente, a espécie está presente em cerca de 15\% (4.000 ha) da área (Martins 2006). Devido a sua agressividade vegetativa impedindo o crescimento de espécies nativas, ampla produção de sementes viáveis e crescimento rápido, o capim-gordura tornouse uma verdadeira ameaça à conservação dos ambientes savânicos e campestres dessa Unidade de Conservação (Funatura/Ibama 1998, Martins et al. 2004).

O objetivo do presente trabalho foi avaliar o impacto da gramínea invasora capim-gordura na biomassa e na riqueza de uma comunidade original de Cerrado sentido restrito (stricto sensu), como também estudar a dinâmica do estrato rasteiro submetido a aplicação de diferentes técnicas de manejo para o controle dessa gramínea.

\section{Material e métodos}

Localização da área de estudo - $\mathrm{O}$ estudo foi conduzido no Parque Nacional de Brasília (PNB) (figura 1), criado pelo Decreto $n^{\circ} 241 / 61$, com cerca de 30.000 ha, situado entre $15^{\circ} 34^{\prime}$ e $15^{\circ} 45^{\prime} \mathrm{S}$ e $48^{\circ} 05^{\prime}$ e $48^{\circ} 53^{\prime} \mathrm{W}$ (Funatura/Ibama 1998). Recentemente, a Lei no 11.282, de 8 de março de 2006 redefiniu sua poligonal, acrescentando cerca de 11.000 ha aos limites noroeste desta Unidade de Conservação. A área experimental, com $2.904 \mathrm{~m}^{2}(44 \mathrm{~m} \times 66 \mathrm{~m})$, insere-se na Zona de Uso Especial e confronta-se com a Zona de Uso Intensivo do Parque. Dista cerca de $400 \mathrm{~m}$ da sede administrativa e cerca de $100 \mathrm{~m}$ da chamada Trilha do Cristal. A vegetação local é definida como Cerrado Ralo, um subtipo fitofisionômico incluso no conceito de Cerrado sentido restrito (sensu Ribeiro \& Walter 2008). Até meados da década de 1990 a área foi usada para o pastejo de cavalos. Segundo Coelho (2002), que elaborou um histórico sobre o regime de incêndios no Parque, a última queimada nesta área ocorreu em 1987.

Coleta e análise dos dados - $\mathrm{O}$ delineamento experimental adotado foi o de blocos ao acaso, com parcelas divididas, quatro repetições, sendo as parcelas os períodos das intervenções ocorridos em maio e setembro, e as subparcelas os tratamentos fogo e manejo integrado, além do controle. As subparcelas tiveram dimensões de $100 \mathrm{~m}^{2}(10 \mathrm{~m} \times 10 \mathrm{~m}) \mathrm{e}$ possuíam uma bordadura de $0,5 \mathrm{~m}$ (figura 2 ).

A primeira técnica de manejo, o tratamento fogo, refere-se à realização de apenas uma queimada controlada (em maio de 2003, antes da floração do capim-gordura; ou em setembro de 2003, após a floração do capim-gordura). $\mathrm{O}$ tratamento manejo integrado consistiu na realização de três intervenções: 1) queimada controlada - maio de 2003, antes da floração do capim-gordura, ou em setembro de 2003, após a floração do capim-gordura; 2) aplicação pontual do herbicida Roundup ${ }^{\circledR}$ (glifosato) em janeiro de 2004 e abril de 2004, sobre as plântulas de capim-gordura e indivíduos dessa gramínea que rebrotaram, e 3) arranquio manual das plântulas de capim-gordura, realizado em janeiro, fevereiro e março de 2005. Dessa forma, os tratamentos avaliados foram controle/maio, controle/setembro, fogo/maio, fogo/setembro, manejo integrado/maio e manejo integrado/setembro.

Nas condições do estudo, para aplicação do herbicida foram gastos em torno de $0,8 \mathrm{~L} \mathrm{ha}^{-1}$. Para realizar esta atividade foi necessário o trabalho de três homens ha ${ }^{-1}$ e o tempo médio para sua execução ficou em torno de 7 horas. Para conduzir o arranquio manual das plântulas do capim gordura (localização, arranquio e retirada da biomassa da área) também foram necessários três homens $\mathrm{ha}^{-1}$, e o tempo médio para realizar esta atividade ficou em torno de 8 horas.

Os levantamentos para determinação da biomassa tiveram início no mês de dezembro de 2002 e se estenderam até maio de 2005 , conforme a seguinte programação de 

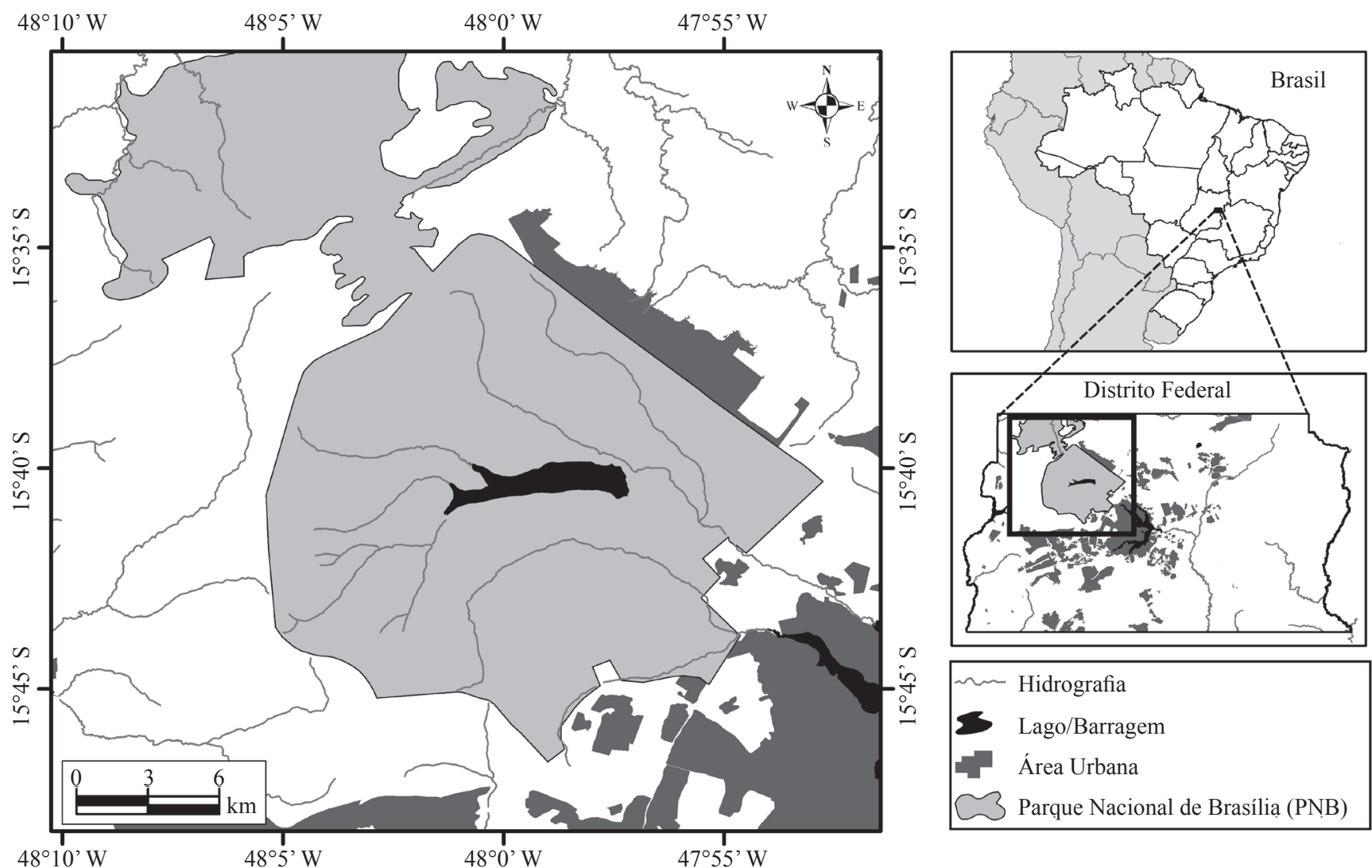

Figura 1. Localização do Parque Nacional de Brasília, Distrito Federal, Brasil.

Figure 1. Localization of the National Park of Brasilia, Federal District, Brazil.

coleta: dezembro de 2002, fevereiro de 2003, abril de 2003, janeiro de 2004, maio de 2004 e maio de 2005. Para o levantamento da biomassa do estrato herbáceo e subarbustivo foram lançados aleatoriamente, em cada repetição de cada tratamento, dez quadrados de $0,25 \mathrm{~m}^{2}(0,50 \mathrm{~m} \times 0,50 \mathrm{~m}) \mathrm{e}$, em cada quadrado, coletou-se toda a biomassa aérea do estrato rasteiro. Posteriormente, o material coletado foi conduzido para o Laboratório de Ecologia da Universidade de Brasília para separação em dois componentes: a) gramíneas nativas + arbustos; e b) capim gordura. Em seguida, todo o material foi seco em estufa a $70^{\circ} \mathrm{C}$, por 48 horas, para determinação do peso seco. Para as avaliações da recuperação da biomassa total e do capim gordura em cada tratamento foi realizado um teste $\mathrm{t}$ para comparações entre as médias do final com as médias antes do início do experimento. As comparações apresentaram erro (b) experimental que se encontra na análise de variância. As comparações entre os valores alcançados pela biomassa total e do capim-gordura entre os tratamentos avaliados (controle/maio, controle/setembro, fogo/maio, fogo/setembro, manejo integrado/maio e manejo integrado/ setembro) no final do experimento foram avaliadas pela análise de variância das parcelas divididas. O programa utilizado foi PROC MIXED do SAS (SAS Institute 2003).
Para o levantamento das espécies, os trabalhos de campo tiveram início em dezembro de 2002 e se estenderam até outubro de 2005. O método adotado para a florística foi o caminhamento, conforme Filgueiras et al. (1994), que consiste em seguir transectos imaginários e anotar todas as espécies ocorrentes ao longo das linhas percorridas. São feitas tantas linhas de caminhada quantas forem necessárias, com o objetivo de se anotar o maior número possível de espécies ocorrentes em cada tratamento. As avaliações de campo foram realizadas semanalmente e o tempo médio para percorrer cada repetição, em cada tratamento, ficou em torno de 20 minutos. Todas as espécies observadas foram coletadas, o que incluiu eventuais espécimes estéreis, utilizados para comparação inicial em herbário. Posteriormente, buscou-se a coleta de espécimes reprodutivos desses espécimes para incorporação em herbário. Os materiais coletados foram herborizados e incorporados nos herbários da Universidade Federal de Uberlândia (HUFU - parte das Asteraceae), Universidade Estadual de Feira de Santana (HUEFS - parte de Mitracarpus e Spermacoce, Rubiaceae), Instituto de Botânica de São de Paulo ( $\mathrm{SP}$ - parte de Leguminosae) e os demais materiais nos Herbários da Universidade de Brasília (UB) e da Embrapa Recursos Genéticos e Biotecnologia (CEN). O tratamento 


$$
\begin{array}{cccc}
\begin{array}{c}
\text { 1o Período } \\
\text { (maio) }
\end{array} & \begin{array}{c}
\text { 2o Período } \\
\text { (setembro) }
\end{array} & \begin{array}{c}
\text { 2o Período } \\
\text { (setembro) }
\end{array} & \begin{array}{c}
\text { 1o Período } \\
\text { (maio) }
\end{array}
\end{array}
$$

\begin{tabular}{|c|c|c|c|}
\hline $\begin{array}{c}\text { Bloco I } \\
\text { Integrado }\end{array}$ & $\begin{array}{c}\text { Bloco I } \\
\text { Fogo }\end{array}$ & $\begin{array}{c}\text { Bloco II } \\
\text { Manejo } \\
\text { Integrado }\end{array}$ & $\begin{array}{c}\text { Bloco II } \\
\text { Controle }\end{array}$ \\
\hline $\begin{array}{c}\text { Bloco I } \\
\text { Controle }\end{array}$ & $\begin{array}{c}\text { Bloco I } \\
\text { Manejo } \\
\text { Integrado }\end{array}$ & $\begin{array}{c}\text { Bloco II } \\
\text { Controle }\end{array}$ & $\begin{array}{c}\text { Bloco II } \\
\text { Fogo }\end{array}$ \\
\hline $\begin{array}{c}\text { Bloco I } \\
\text { Fogo }\end{array}$ & $\begin{array}{c}\text { Bloco I } \\
\text { Controle }\end{array}$ & $\begin{array}{c}\text { Bloco II } \\
\text { Fogo }\end{array}$ & $\begin{array}{c}\text { Bloco II } \\
\text { Manejo } \\
\text { Integrado }\end{array}$ \\
\hline $\begin{array}{c}\text { Bloco III } \\
\text { Manejo } \\
\text { Integrado }\end{array}$ & $\begin{array}{c}\text { Bloco III } \\
\text { Fogo } \\
\text { Bloco IV } \\
\text { Controle }\end{array}$ & $\begin{array}{c}\text { Bloco IV } \\
\text { Controle }\end{array}$ \\
\hline $\begin{array}{c}\text { Bloco III } \\
\text { Fogo }\end{array}$ & $\begin{array}{c}\text { Bloco III } \\
\text { Controle }\end{array}$ & $\begin{array}{c}\text { Bloco IV } \\
\text { Fogo }\end{array}$ & $\begin{array}{c}\text { Bloco IV } \\
\text { Manejo } \\
\text { Integrado }\end{array}$ \\
\hline $\begin{array}{c}\text { Bloco III } \\
\text { Controle }\end{array}$ & $\begin{array}{c}\text { Bloco III } \\
\text { Manejo } \\
\text { Integrado }\end{array}$ & $\begin{array}{c}\text { Bloco IV } \\
\text { Manejo } \\
\text { Integrado }\end{array}$ & $\begin{array}{c}\text { Bloco IV } \\
\text { Fogo }\end{array}$ \\
\hline $\begin{array}{c}\text { 2o Período } \\
\text { (setembro) }\end{array}$ & $\begin{array}{c}\text { 1o Período } \\
\text { (maio) }\end{array}$ & $\begin{array}{c}\text { 2o Período } \\
\text { (setembro) }\end{array}$ & $\begin{array}{c}\text { 1o Período } \\
\text { (maio) }\end{array}$ \\
\hline
\end{tabular}

Figura 2. Delineamento experimental onde os períodos das intervenções (maio e setembro) são as parcelas (separadas por linhas mais grossas) e os tratamentos (controle, fogo e manejo integrado) as subparcelas (separadas por linhas mais finas). Cada subparcela corresponde a $100 \mathrm{~m}^{2}$. Há uma zona tampão de $0,5 \mathrm{~m}$ entre os tratamentos. taxonômico de famílias seguiu o APG II (2003), com base em Souza \& Lorenzi (2008).

\section{Resultados e discussão}

$\mathrm{Na}$ área experimental foram registradas 402 espécies, distribuídas em 213 gêneros e 60 famílias (tabela 1), das quais uma única foi de Pteridófita (Schizaeaceae, com a espécie Anemia pastinacaria). As famílias mais representativas foram Asteraceae (68 espécies), Poaceae (53 espécies), Leguminosae (50 espécies), Malpighiaceae (20 espécies), Myrtaceae (18 espécies) e Lamiaceae (16 espécies). Estas seis famílias, que representam apenas $10 \%$ das famílias amostradas, concentraram 55,97\% do total de espécies. Essa característica de concentração das espécies em poucas famílias tem sido relatada frequentemente em ecossistemas tropicais, e no bioma Cerrado em particular (Tannus \& Assis 2004, Munhoz \& Felfili 2006, 2008, Ishara et al. 2008, Pinto et al. 2009).

Nos tratamentos estudados foram anotados os seguintes números de espécies: controle/maio (260 espécies); controle/setembro (245 espécies); fogo/maio (258 espécies); fogo/setembro (271 espécies); manejo integrado/maio (268 espécies) e manejo integrado/ setembro (309 espécies). Estes números revelam que,

Figure 2. Experimental design where the periods of the interventions (May and September) are plots (separated by thick lines) and the treatments (control, fire and integrated management) are the subplots (separated by thin lines). Each subparcel corresponds to $100 \mathrm{~m}^{2}$. There is a $0.5 \mathrm{~m}$ buffer zone between treatment.

Tabela 1. Espécies amostradas ao longo de três anos na área experimental do Parque Nacional de Brasília, Brasília, DF. Ordem alfabética de famílias (APG II 2003). Freq = frequência das espécies na área experimental ( $n=24$ tratamentos) e ocorrência das espécies nos diferentes tratamentos: $\mathrm{CM}=$ controle maio (4 tratamentos); $\mathrm{CS}=$ controle setembro (4 tratamentos); $\mathrm{FM}=$ fogo maio (4 tratamentos); FS = fogo setembro (4 tratamentos); MIM = manejo integrado maio (4 tratamentos); e MIS = manejo integrado setembro (4 tratamentos). Espécies exóticas indicadas por asterisco (Mendonça et al. 2008). CRM = Coleção de Carlos Romero Martins.

Table 1. Species collected over three years of study in an experimental area in the National Park of Brasilia, Brasilia, DF. Families are presented in alphabetical order (APG II, 2003). Freq. = frequency of the species in the experimental area $(n=24$ plots $)$ and occurrence of the species in the different treatments: $\mathrm{CM}=$ control May (4 plots); $\mathrm{CS}=$ control September (4 plots); FM = fire May (4 plots); FS = fire September (4 plots); MIM = integrated management May (4 plots); and MIS = integrated management September (4 plots). Exotic species indicated by asterisk (Mendonça et al. 2008). CRM = Collection of Carlos Romero Martins.

\begin{tabular}{lllllllll}
\hline Família/Espécie & Hábito & Freq & CM & CS & FM & FS & MIM & MIS \\
\hline ACANTHACEAE & & & & & & & & \\
Justicia oncoides (Lindau) Wassh. \& C. Ezcurra & Subarbusto & 54 & x & x & x & x & x & x \\
Justicia pycnophylla Lindau & Subarbusto & 70 & x & x & x & x & x & x \\
Ruellia brevicaulis (Nees) Lindau & Subarbusto & 12 & & & & x & x & x \\
& & & & & & & continua
\end{tabular}


continuação

\begin{tabular}{|c|c|c|c|c|c|c|c|c|}
\hline Família/Espécie & Hábito & Freq $^{1}$ & $\mathrm{CM}$ & $\mathrm{CS}$ & FM & FS & MIM & MIS \\
\hline Ruellia geminiflora Kunth & Erva & 50 & $\mathrm{x}$ & $\mathrm{x}$ & $\mathrm{x}$ & $\mathrm{x}$ & $\mathrm{x}$ & $\mathrm{x}$ \\
\hline Ruellia sp. (CRM 301) & Subarbusto & 4 & & & & & $\mathrm{x}$ & \\
\hline \multicolumn{9}{|l|}{ ALSTROEMERIACEAE } \\
\hline Alstroemeria punctata Ravenna & Erva & 25 & & $\mathrm{x}$ & $\mathrm{x}$ & $\mathrm{x}$ & & $\mathrm{x}$ \\
\hline \multicolumn{9}{|l|}{ ANACARDIACEAE } \\
\hline Anacardium humile A. St.-Hil. & Arbusto & 37 & $\mathrm{x}$ & $\mathrm{x}$ & $\mathrm{x}$ & $\mathrm{x}$ & $\mathrm{x}$ & $\mathrm{x}$ \\
\hline Schinus terebenthifolius Raddi & Árvore & 62 & $\mathrm{x}$ & $\mathrm{x}$ & $\mathrm{x}$ & $\mathrm{x}$ & $\mathrm{x}$ & $\mathrm{x}$ \\
\hline \multicolumn{9}{|l|}{ ANNONACEAE } \\
\hline Annona monticola Mart. & Arbusto & 83 & $\mathrm{x}$ & $\mathrm{x}$ & $\mathrm{x}$ & $\mathrm{x}$ & $\mathrm{x}$ & $\mathrm{x}$ \\
\hline Annona tomentosa R.E. Fr. & Arbusto & 62 & $\mathrm{x}$ & $\mathrm{x}$ & $\mathrm{x}$ & $\mathrm{x}$ & $\mathrm{x}$ & $\mathrm{x}$ \\
\hline \multicolumn{9}{|l|}{ APIACEAE } \\
\hline Eryngium juncifolium (Urb.) Mathias \& Constance & Erva & 29 & $\mathrm{x}$ & $\mathrm{x}$ & & $\mathrm{x}$ & & $\mathrm{x}$ \\
\hline \multicolumn{9}{|l|}{ APOCYNACEAE } \\
\hline Asclepias candida Vell. & Erva & 8 & & & $\mathrm{x}$ & $\mathrm{x}$ & & \\
\hline Barjonia erecta (Vell.) K. Schum. & Subarbusto & 25 & & $\mathrm{x}$ & $\mathrm{x}$ & & $\mathrm{x}$ & \\
\hline Barjonia glazioui Marquete & Subarbusto & 50 & $\mathrm{x}$ & $\mathrm{x}$ & $\mathrm{x}$ & $\mathrm{x}$ & $\mathrm{x}$ & $\mathrm{x}$ \\
\hline Blephrarodon nitidum (Vell.) J. F. Macbr. & Trepadeira & 16 & & $\mathrm{x}$ & $\mathrm{x}$ & & $\mathrm{x}$ & \\
\hline Himatanthus obovatus (Müll. Arg) Woods. & Árvore & 25 & $\mathrm{x}$ & $\mathrm{x}$ & $\mathrm{x}$ & $\mathrm{x}$ & & $\mathrm{x}$ \\
\hline Mandevilla pohliana (Stadelm.) A. H. Gentry & Subarbusto & 8 & $\mathrm{x}$ & & & & & $\mathrm{x}$ \\
\hline Oxypetalum capitatum Mart. \& Zucc. & Erva & 20 & $\mathrm{x}$ & & & $\mathrm{x}$ & $\mathrm{x}$ & $\mathrm{x}$ \\
\hline Oxypetalum erectum Mart. \& Zucc. & Subarbusto, Arbusto & 8 & & & & & $\mathrm{x}$ & $\mathrm{x}$ \\
\hline Tabernaemontana catharinensis A. DC. & Arbusto & 91 & $\mathrm{x}$ & $\mathrm{x}$ & $\mathrm{x}$ & $\mathrm{x}$ & $\mathrm{x}$ & $\mathrm{x}$ \\
\hline \multicolumn{9}{|l|}{ ARECACEAE } \\
\hline Allagoptera campestris (Mart.) Kuntze & Palmeira acaule & 16 & $\mathrm{x}$ & & & $\mathrm{x}$ & $\mathrm{x}$ & \\
\hline Butia archeri (Glassman) Glassman & Palmeira arbustiva & 41 & $\mathrm{x}$ & $\mathrm{x}$ & $\mathrm{x}$ & $\mathrm{x}$ & $\mathrm{x}$ & $\mathrm{x}$ \\
\hline Syagrus comosa (Mart.) Mart. & Palmeira arbustiva & 4 & $\mathrm{x}$ & & & & & \\
\hline Syagrus flexuosa (Mart.) Becc. & Palmeira arbustiva & 29 & $\mathrm{x}$ & & $\mathrm{x}$ & & $\mathrm{x}$ & $\mathrm{x}$ \\
\hline \multicolumn{9}{|l|}{ ASTERACEAE } \\
\hline Acanthospermum australe (Loefl.) Kuntze* & Erva & 50 & $\mathrm{x}$ & $\mathrm{x}$ & $\mathrm{x}$ & $\mathrm{x}$ & $\mathrm{x}$ & $\mathrm{x}$ \\
\hline Achyrocline satureioides (Lam.) A. DC. & Erva & 87 & $\mathrm{x}$ & $\mathrm{x}$ & $\mathrm{x}$ & $\mathrm{x}$ & $\mathrm{x}$ & $\mathrm{x}$ \\
\hline Ageratum conyzoides L.* & Erva & 25 & & & $\mathrm{x}$ & & $\mathrm{x}$ & $\mathrm{x}$ \\
\hline Ageratum fastigiatum (Gardner) R. M. King \& H. Rob. & Subarbusto & 12 & $\mathrm{x}$ & & & $\mathrm{x}$ & & $\mathrm{x}$ \\
\hline $\begin{array}{l}\text { Ageratum myriadenium (Sch. Bip. ex Baker) R. M. } \\
\text { King \& H. Rob. }\end{array}$ & Erva & 20 & $\mathrm{x}$ & $\mathrm{x}$ & $\mathrm{x}$ & $\mathrm{x}$ & & \\
\hline Aspilia riedelii Baker & Subarbusto & 50 & $\mathrm{x}$ & $\mathrm{x}$ & $\mathrm{x}$ & $\mathrm{x}$ & $\mathrm{x}$ & $\mathrm{x}$ \\
\hline Ayapana amygdalina (Lam.) R. M. King \& H. Rob. & Subarbusto & 16 & & $\mathrm{x}$ & $\mathrm{x}$ & $\mathrm{x}$ & $\mathrm{x}$ & \\
\hline Baccharis dracunculifolia A. DC. & Arbusto & 91 & $\mathrm{x}$ & $\mathrm{x}$ & $\mathrm{x}$ & $\mathrm{x}$ & $\mathrm{x}$ & $\mathrm{x}$ \\
\hline Baccharis humilis Sch. Bip. ex Baker & Erva & 16 & & & & $\mathrm{x}$ & & $\mathrm{x}$ \\
\hline Baccharis reticularia A. DC & Arbusto & 4 & & $\mathrm{x}$ & & & & \\
\hline Baccharis subdentata A. DC. & Erva & 45 & $\mathrm{x}$ & $\mathrm{x}$ & $\mathrm{x}$ & $\mathrm{x}$ & $\mathrm{x}$ & $\mathrm{x}$ \\
\hline Bidens graveolens Mart. & Arbusto & 62 & $\mathrm{x}$ & $\mathrm{x}$ & $\mathrm{x}$ & $\mathrm{x}$ & $\mathrm{x}$ & $\mathrm{x}$ \\
\hline Bidens pilosa L. * & Erva & 33 & $\mathrm{x}$ & $\mathrm{x}$ & & $\mathrm{x}$ & $\mathrm{x}$ & $\mathrm{x}$ \\
\hline Blainvillea dichotoma (Murray) Stewart & Erva & 4 & & & & & & $\mathrm{x}$ \\
\hline Calea reticulata Gardner & Subarbusto & 4 & & & & & & $\mathrm{x}$ \\
\hline $\begin{array}{l}\text { Campuloclinium megacephalum (Mart. ex Baker) R. } \\
\text { M. King \& H. Rob. }\end{array}$ & Subarbusto & 12 & $\mathrm{x}$ & & & $\mathrm{x}$ & & $\mathrm{x}$ \\
\hline Chaptalia integerrima (Vell.) Burkart & Erva & 37 & $\mathrm{x}$ & & $\mathrm{x}$ & $\mathrm{x}$ & $\mathrm{x}$ & $\mathrm{x}$ \\
\hline Chaptalia nutans (L.) Polak * & Erva & 8 & & $\mathrm{x}$ & & $\mathrm{x}$ & & \\
\hline Chresta sphaerocephala DC. & Arbusto & 8 & & & & $\mathrm{x}$ & & $\mathrm{x}$ \\
\hline $\begin{array}{l}\text { Chromolaena chaseae (B. L. Rob.) R. M. King \& H. } \\
\text { Rob. }\end{array}$ & Subarbusto & 45 & $\mathrm{x}$ & $\mathrm{x}$ & $\mathrm{x}$ & $\mathrm{x}$ & $\mathrm{x}$ & $\mathrm{x}$ \\
\hline $\begin{array}{l}\text { Chromolaena laevigata (Lam.) R. M. King \& H. Rob. } \\
\text { (=Eupatorium laevigatum Lam.) * }\end{array}$ & Subarbusto, arbusto & 41 & $\mathrm{x}$ & $\mathrm{x}$ & $\mathrm{x}$ & $\mathrm{x}$ & $\mathrm{x}$ & \\
\hline Chromolaena leucocephala Gardner & Arbusto & 8 & $\mathrm{x}$ & $\mathrm{x}$ & & & & \\
\hline
\end{tabular}


continuação

\begin{tabular}{|c|c|c|c|c|c|c|c|c|}
\hline Família/Espécie & Hábito & Freq $^{1}$ & $\mathrm{CM}$ & $\mathrm{CS}$ & FM & FS & MIM & MIS \\
\hline $\begin{array}{l}\text { Chromolaena maximilianii (Schrad. ex DC.) R. M. King } \\
\text { \& H. Rob. (=Eupatorium maximiliani Schrad. ex DC.)* }\end{array}$ & Subarbusto, arbusto & 20 & & $\mathrm{x}$ & & $\mathrm{x}$ & $\mathrm{x}$ & $\mathrm{x}$ \\
\hline $\begin{array}{l}\text { Chromolaena } \mathrm{cf} \text {. myriocephala (Gardner) R. M. King } \\
\text { \& H. Rob. (=Eupatorium myriocephalum Gardner) }\end{array}$ & Erva & 8 & & $\mathrm{x}$ & & & & $\mathrm{x}$ \\
\hline $\begin{array}{l}\text { Chromolaena stachyophylla (Spreng.) R. M. King \& } \\
\text { H. Rob. (=Eupatorium stachyophyllum Spreng.) }\end{array}$ & Erva, subarbusto & 20 & $\mathrm{x}$ & $\mathrm{x}$ & & & $\mathrm{x}$ & $\mathrm{x}$ \\
\hline $\begin{array}{l}\text { Chrysolaena herbacea (Vell.) H. Rob. (=Vernonia } \\
\text { herbacea (Vell.) Rusby) }\end{array}$ & Erva & 75 & $\mathrm{x}$ & $\mathrm{x}$ & $\mathrm{x}$ & $\mathrm{x}$ & $\mathrm{x}$ & $\mathrm{x}$ \\
\hline Conyza canadensis (L.) Cronquist* & Erva & 25 & $\mathrm{x}$ & & & $\mathrm{x}$ & $\mathrm{x}$ & $\mathrm{x}$ \\
\hline Dimerostemma lippioides (Baker) S. F. Blake & Erva & 16 & $\mathrm{x}$ & $\mathrm{x}$ & $\mathrm{x}$ & & & \\
\hline Disynaphia halimifolia (DC.) R. M. King \& H. Rob. & Subarbusto & 58 & & & $\mathrm{x}$ & $\mathrm{x}$ & $\mathrm{x}$ & $\mathrm{x}$ \\
\hline $\begin{array}{l}\text { Echinocoryne holosericea (Mart. ex DC.) H. Rob. } \\
\text { (=Vernonia holosericea Mart. ex DC.) }\end{array}$ & Arbusto & 33 & $\mathrm{x}$ & $\mathrm{x}$ & $\mathrm{x}$ & $\mathrm{x}$ & & $\mathrm{x}$ \\
\hline Elephantopus biflorus (Less.) Sch. Bip & Subarbusto & 8 & & & & & $\mathrm{x}$ & $\mathrm{x}$ \\
\hline Elephantopus elongatus Gardner & Arbusto & 33 & $\mathrm{x}$ & & $\mathrm{x}$ & $\mathrm{x}$ & $\mathrm{x}$ & $\mathrm{x}$ \\
\hline Elephantopus mollis Kunth* & Erva & 75 & $\mathrm{x}$ & $\mathrm{x}$ & $\mathrm{x}$ & $\mathrm{x}$ & $\mathrm{x}$ & $\mathrm{x}$ \\
\hline Emilia sonchifolia (L.) DC * & Erva & 4 & & & & & & $\mathrm{x}$ \\
\hline Erechtites hieraciifolia (L.) Raf. ex DC. & Erva & 8 & & & $\mathrm{x}$ & & & \\
\hline $\begin{array}{l}\text { Eremanthus mollis Sch. Bip. (=Vernonia pannosa } \\
\text { (Baker) MacLeish) }\end{array}$ & Subarbusto, arbusto & 8 & & & $\mathrm{x}$ & & & \\
\hline Ichthyothere latifolia Baker & Erva & 100 & $\mathrm{x}$ & $\mathrm{x}$ & $\mathrm{x}$ & $\mathrm{x}$ & $\mathrm{x}$ & $\mathrm{x}$ \\
\hline $\begin{array}{l}\text { Lepidaploa aurea (Mart. ex DC.) H. Rob. (=Vernonia } \\
\text { aurea Mart. ex DC.) }\end{array}$ & Arbusto & 75 & $\mathrm{x}$ & $\mathrm{x}$ & $\mathrm{x}$ & $\mathrm{x}$ & $\mathrm{x}$ & $\mathrm{x}$ \\
\hline $\begin{array}{l}\text { Lessingianthus argyrophyllus (Less.) H. Rob. } \\
\text { (=Vernonia argyrophylla Less.) }\end{array}$ & Subarbusto & 4 & & $\mathrm{x}$ & $\mathrm{x}$ & $\mathrm{x}$ & $\mathrm{x}$ & $\mathrm{x}$ \\
\hline $\begin{array}{l}\text { Lessingianthus bardanoides (Less.) H. Rob. } \\
\text { (=Vernonia bardanoides Less.) }\end{array}$ & Arbusto & 20 & $\mathrm{x}$ & & & $\mathrm{x}$ & $\mathrm{x}$ & $\mathrm{x}$ \\
\hline $\begin{array}{l}\text { Lessingianthus buddleiifolius (Mart. ex DC.) H.Rob. } \\
\text { (=Vernonia buddleiilifolia Mart. ex DC.) }\end{array}$ & Arbusto & 33 & $\mathrm{x}$ & & $\mathrm{x}$ & $\mathrm{x}$ & $\mathrm{x}$ & $\mathrm{x}$ \\
\hline $\begin{array}{l}\text { Lessingianthus durus (Mart. ex DC.) H. Rob. } \\
\text { (=Vernonia dura Mart. ex DC.) }\end{array}$ & Subarbusto & 8 & & & & $\mathrm{x}$ & & $\mathrm{x}$ \\
\hline $\begin{array}{l}\text { Lessingianthus elegans (Gardner) H. Rob. (=Vernonia } \\
\text { elegans Gardner) }\end{array}$ & Subarbusto & 4 & & & & & & $\mathrm{x}$ \\
\hline $\begin{array}{l}\text { Lessingianthus irwinii (G. M. Barroso) H. Rob. } \\
\text { (=Vernonia irwinii G. M Barroso) }\end{array}$ & Subarbusto & 25 & $\mathrm{x}$ & & $\mathrm{x}$ & $\mathrm{x}$ & $\mathrm{x}$ & \\
\hline $\begin{array}{l}\text { Lessingianthus ligulifolius (Mart. ex DC.) H. Rob. } \\
\text { (=Vernonia ligulifolia Mart. ex DC.) }\end{array}$ & Erva & 12 & & & $\mathrm{x}$ & & & $\mathrm{x}$ \\
\hline $\begin{array}{l}\text { Lessingianthus simplex (Less.) H. Rob. (=Vernonia } \\
\text { simplex Less.) }\end{array}$ & Erva & 12 & & & $\mathrm{x}$ & & $\mathrm{x}$ & \\
\hline Mikania cordifolia (L. f.) Willd. & Trepadeira & 16 & $\mathrm{x}$ & $\mathrm{x}$ & & & & $\mathrm{x}$ \\
\hline Podocoma bellidifolia Baker & Erva & 16 & & & & & $\mathrm{x}$ & $\mathrm{x}$ \\
\hline Podocoma hieracifolia (Poir.) Cass. & Subarbusto & 20 & $\mathrm{x}$ & & & & $\mathrm{x}$ & $\mathrm{x}$ \\
\hline Porophyllum angustissimum Gardner & Arbusto & 20 & $\mathrm{x}$ & & $\mathrm{x}$ & & $\mathrm{x}$ & $\mathrm{x}$ \\
\hline Porophyllum ruderale (Jacq.) Cass. * & Erva & 16 & $\mathrm{x}$ & & $\mathrm{x}$ & & & $\mathrm{x}$ \\
\hline $\begin{array}{l}\text { Praxelis clematidea (Griseb.) R. M. King \& H. Rob. } \\
\text { (=Eupatorium clematideum Griseb.) }\end{array}$ & Erva & 33 & $\mathrm{x}$ & & $\mathrm{x}$ & & $\mathrm{x}$ & $\mathrm{x}$ \\
\hline Pterocaulon lanatum Kuntze & Subarbusto & 4 & & & $\mathrm{x}$ & & & \\
\hline $\begin{array}{l}\text { Pterocaulon rugosum }(\text { Vahl) Malme (=Pterocaulon } \\
\text { alopecuroides (Lam.) DC.) }\end{array}$ & Subarbusto & 8 & & & $\mathrm{x}$ & & $\mathrm{x}$ & \\
\hline Riencourtia oblongifolia Gardner & Subarbusto & 16 & & & $\mathrm{x}$ & $\mathrm{x}$ & $\mathrm{x}$ & \\
\hline Sonchus oleraceus L. * & Erva & 20 & & & $\mathrm{x}$ & $\mathrm{x}$ & $\mathrm{x}$ & $\mathrm{x}$ \\
\hline $\begin{array}{l}\text { Stenocephalum apiculatum (Mart. ex DC.) Sch. Bip. } \\
\text { (=Vernonia apiculata Mart. ex DC.) }\end{array}$ & Erva & 33 & $\mathrm{x}$ & & & $\mathrm{x}$ & & \\
\hline Stenophalium heringeri (H. Rob.) Anderb. & Erva & 75 & $\mathrm{x}$ & $\mathrm{x}$ & $\mathrm{x}$ & $\mathrm{x}$ & $\mathrm{x}$ & $\underset{\mathrm{x}}{\mathrm{x}}$ \\
\hline
\end{tabular}


continuação

\begin{tabular}{|c|c|c|c|c|c|c|c|c|}
\hline Família/Espécie & Hábito & Freq $^{1}$ & $\mathrm{CM}$ & $\mathrm{CS}$ & FM & FS & MIM & MIS \\
\hline Stevia heptachaeta DC. & Erva & 29 & $\mathrm{x}$ & $\mathrm{x}$ & $\mathrm{x}$ & $\mathrm{x}$ & $\mathrm{x}$ & $\mathrm{x}$ \\
\hline $\begin{array}{l}\text { Stomatanthes trigonus (Gardner) H. Rob. } \\
\text { (=Eupatorium trigonum Gardner) }\end{array}$ & Arbusto & 8 & $\mathrm{x}$ & & & & & $\mathrm{x}$ \\
\hline Tagetes minuta L. * & Erva & 8 & & & & & $\mathrm{x}$ & $\mathrm{x}$ \\
\hline Trichogonia salviaefolia Gardner & Erva, subarbusto & 96 & $\mathrm{x}$ & $\mathrm{x}$ & $\mathrm{x}$ & $\mathrm{x}$ & $\mathrm{x}$ & $\mathrm{x}$ \\
\hline Tridax procumbens L. * & Erva & 12 & & & $\mathrm{x}$ & & & $\mathrm{x}$ \\
\hline Trixis glutinosa D. Don & Subarbusto & 16 & & & & $\mathrm{x}$ & $\mathrm{x}$ & $\mathrm{x}$ \\
\hline $\begin{array}{l}\text { Vernonanthura ferruginea (Less.) H. Rob. } \\
\text { (=Vernonia ferruginea Less.) }\end{array}$ & Arbusto & 25 & $\mathrm{x}$ & $\mathrm{x}$ & & $\mathrm{x}$ & $\mathrm{x}$ & $\mathrm{x}$ \\
\hline Vernonia rubriramea Mart. ex DC. & Arbusto & 20 & & & $\mathrm{x}$ & $\mathrm{x}$ & $\mathrm{x}$ & $\mathrm{x}$ \\
\hline Viguiera robusta Gardner & Erva & 12 & $\mathrm{x}$ & & & $\mathrm{x}$ & & $\mathrm{x}$ \\
\hline \multicolumn{9}{|l|}{ BIGNONIACEAE } \\
\hline Anemopaegma arvense (Vell.) Stellfeld ex de Souza & Subarbusto & 79 & $\mathrm{x}$ & $\mathrm{x}$ & $\mathrm{x}$ & $\mathrm{x}$ & $\mathrm{x}$ & $\mathrm{x}$ \\
\hline Arrabidea brachypoda (DC.) Bureau & Arbusto & 12 & $\mathrm{x}$ & & & $\mathrm{x}$ & & \\
\hline Jacaranda caroba (Vell.) DC. & Árvore & 4 & & & & $\mathrm{x}$ & & \\
\hline Jacaranda ulei Bureau \& K. Schum. & Subarbusto & 79 & $\mathrm{x}$ & $\mathrm{x}$ & $\mathrm{x}$ & $\mathrm{x}$ & $\mathrm{x}$ & $\mathrm{x}$ \\
\hline Tabebuia aurea (Manso) Benth. \& Hook. f. ex S. Moore & Árvore & 8 & & & & $\mathrm{x}$ & & $\mathrm{x}$ \\
\hline Tabebuia ochracea (Cham.) Standl. & Árvore & 83 & $\mathrm{x}$ & $\mathrm{x}$ & $\mathrm{x}$ & $\mathrm{x}$ & $\mathrm{x}$ & $\mathrm{x}$ \\
\hline Zeyheria montana Mart. & Arbusto & 25 & $\mathrm{x}$ & & $\mathrm{x}$ & $\mathrm{x}$ & $\mathrm{x}$ & $\mathrm{x}$ \\
\hline \multicolumn{9}{|l|}{ BORAGINACEAE } \\
\hline Cordia corymbosa (L.) G. Don & Arbusto & 16 & $\mathrm{x}$ & $\mathrm{x}$ & & & & \\
\hline Cordia truncata Fresen. & Arbusto & 58 & $\mathrm{x}$ & $\mathrm{x}$ & $\mathrm{x}$ & $\mathrm{x}$ & $\mathrm{x}$ & $\mathrm{x}$ \\
\hline \multicolumn{9}{|l|}{ BROMELIACEAE } \\
\hline Dyckia brasiliana L.B. Sm. & Erva & 25 & $\mathrm{x}$ & & $\mathrm{x}$ & $\mathrm{x}$ & $\mathrm{x}$ & $\mathrm{x}$ \\
\hline \multicolumn{9}{|l|}{ CANNABACEAE } \\
\hline Trema micrantha (L.) Blume & Árvore & 4 & & & & $\mathrm{x}$ & & \\
\hline \multicolumn{9}{|l|}{ CARYOCARACEAE } \\
\hline Caryocar brasiliense Cambess. & Árvore & 25 & $\mathrm{x}$ & $\mathrm{x}$ & $\mathrm{x}$ & $\mathrm{x}$ & $\mathrm{x}$ & \\
\hline \multicolumn{9}{|l|}{ CELASTRACEAE } \\
\hline Peritassa campestris (Cambess.) A. C. Sm. & Arbusto & 4 & & & & & $\mathrm{x}$ & \\
\hline Plenckia populnea Reissek & Árvore & 8 & & $\mathrm{x}$ & & & & $\mathrm{x}$ \\
\hline Tontelea micrantha (Mart. ex Schult.) A. C. Sm. & Arbusto & 67 & $\mathrm{x}$ & $\mathrm{x}$ & $\mathrm{x}$ & $\mathrm{x}$ & $\mathrm{x}$ & $\mathrm{x}$ \\
\hline \multicolumn{9}{|l|}{ CHRYSOBALANACEAE } \\
\hline Parinari obtusifolia Hook. f. & Arbusto & 50 & & $\mathrm{x}$ & $\mathrm{x}$ & $\mathrm{x}$ & $\mathrm{x}$ & $\mathrm{x}$ \\
\hline \multicolumn{9}{|l|}{ CLUSIACEAE } \\
\hline Kielmeyera abdita Saddi & Arbusto & 41 & $\mathrm{x}$ & $\mathrm{x}$ & $\mathrm{x}$ & $\mathrm{x}$ & $\mathrm{x}$ & $\mathrm{x}$ \\
\hline Kielmeyera coriacea Mart. \& Zucc. & Árvore & 62 & $\mathrm{x}$ & $\mathrm{x}$ & $\mathrm{x}$ & $\mathrm{x}$ & $\mathrm{x}$ & $\mathrm{x}$ \\
\hline Kielmeyera variabilis Mart. & Subarbusto & 4 & & & & $\mathrm{x}$ & & \\
\hline \multicolumn{9}{|l|}{ CONNARACEAE } \\
\hline Connarus suberosus Planch. & Árvore & 4 & & & & & $\mathrm{x}$ & \\
\hline \multicolumn{9}{|l|}{ CONVOVULACEAE } \\
\hline Evolvulus gypsophiloides Moric. & Subarbusto & 75 & $\mathrm{x}$ & $\mathrm{x}$ & $\mathrm{x}$ & $\mathrm{x}$ & $\mathrm{x}$ & $\mathrm{x}$ \\
\hline Evolvulus lagopodioides Meisn. & Erva & 75 & & & & $\mathrm{x}$ & & $\mathrm{x}$ \\
\hline Ipomoea procumbens Mart. \& Choisy & Trepadeira & 4 & $\mathrm{x}$ & & & & & \\
\hline $\begin{array}{l}\text { Ipomoea stenophylla Meissn. (=Ipomoea aurifolia } \\
\text { Dammer) }\end{array}$ & Subarbusto & 25 & & & $\mathrm{x}$ & & $\mathrm{x}$ & $\mathrm{x}$ \\
\hline Merremia digitata (Spreng.) Hallier f. & Erva & 16 & $\mathrm{x}$ & $\mathrm{x}$ & & & $\mathrm{x}$ & \\
\hline Merremia tomentosa (Choisy) Hallier f. & Subarbusto & 12 & & & $\mathrm{x}$ & & $\mathrm{x}$ & $\mathrm{x}$ \\
\hline \multicolumn{9}{|l|}{ CUCURBITACEAE } \\
\hline Melancium campestre Naudin & Trepadeira & 8 & & & & & & $\mathrm{x}$ \\
\hline \multicolumn{9}{|l|}{ CYPERACEAE } \\
\hline Bulbostylis capillaris (L.) C. B. Clarke & Erva & 8 & & & $\mathrm{x}$ & & & $\mathrm{x}$ \\
\hline $\begin{array}{l}\text { Bulbostylis junciformis (Kunth) C. B. Clarke ex } \\
\text { S. Moore }\end{array}$ & Erva & 20 & & $\mathrm{x}$ & $\mathrm{x}$ & $\mathrm{x}$ & $x$ & \\
\hline
\end{tabular}


continuação

\begin{tabular}{|c|c|c|c|c|c|c|c|c|}
\hline Família/Espécie & Hábito & Freq $^{1}$ & $\mathrm{CM}$ & $\mathrm{CS}$ & FM & FS & MIM & MIS \\
\hline Bulbostylis sp. (CRM 287) & Erva & 20 & & $\mathrm{x}$ & $\mathrm{x}$ & $\mathrm{x}$ & & \\
\hline Rhynchospora consanguinea (Kunth) Böckeler & Erva & 45 & $\mathrm{x}$ & $\mathrm{x}$ & $\mathrm{x}$ & $\mathrm{x}$ & $\mathrm{x}$ & $\mathrm{x}$ \\
\hline Rhyncospora rugosa (Vahl) Gale & Erva & 12 & & & $\mathrm{x}$ & & $\mathrm{x}$ & \\
\hline Scleria scabra Willd. & Erva & 29 & $\mathrm{x}$ & $\mathrm{x}$ & & $\mathrm{x}$ & $\mathrm{x}$ & $\mathrm{x}$ \\
\hline \multicolumn{9}{|l|}{ DILLENIACEAE } \\
\hline Davilla elliptica A. St.-Hil. & Arbusto & 75 & $\mathrm{x}$ & $\mathrm{x}$ & $\mathrm{x}$ & $\mathrm{x}$ & $\mathrm{x}$ & $\mathrm{x}$ \\
\hline \multicolumn{9}{|l|}{ ERYTHROXYLACEAE } \\
\hline Erythroxylum amplifolium (Mart.) O. E. Schulz & Árvore & 50 & $\mathrm{x}$ & & $\mathrm{x}$ & $\mathrm{x}$ & $\mathrm{x}$ & $\mathrm{x}$ \\
\hline Erythroxylum campestre A. St.-Hil. & Subarbusto & 96 & $\mathrm{x}$ & $\mathrm{x}$ & $\mathrm{x}$ & $\mathrm{x}$ & $\mathrm{x}$ & $\mathrm{x}$ \\
\hline Erythroxylum nanum A. St.-Hil. & Subarbusto & 75 & $\mathrm{x}$ & $\mathrm{x}$ & $\mathrm{x}$ & $\mathrm{x}$ & $\mathrm{x}$ & $\mathrm{x}$ \\
\hline Erythroxylum suberosum A. St.-Hil. & Arbusto & 4 & & & & & & $\mathrm{x}$ \\
\hline Erythroxylum tortuosum Mart. & Árvore & 4 & & & $\mathrm{x}$ & & & \\
\hline \multicolumn{9}{|l|}{ EUPHORBIACEAE } \\
\hline Acalypha claussenii (Turcz.) Müll. Arg. & Erva & 29 & $\mathrm{x}$ & & $\mathrm{x}$ & $\mathrm{x}$ & $\mathrm{x}$ & $\mathrm{x}$ \\
\hline Chamaesyce potentilloides (Boiss.) Croizat & Erva & 8 & & & $\mathrm{x}$ & $\mathrm{x}$ & & \\
\hline Croton antisyphiliticus Mart. & Erva & 91 & $\mathrm{x}$ & $\mathrm{x}$ & $\mathrm{x}$ & $\mathrm{x}$ & $\mathrm{x}$ & $\mathrm{x}$ \\
\hline Croton campestris A. St.-Hil. & Arbusto & 58 & $\mathrm{x}$ & $\mathrm{x}$ & $\mathrm{x}$ & $\mathrm{x}$ & $\mathrm{x}$ & $\mathrm{x}$ \\
\hline Croton didrichsenii G. L.Webster & Subarbusto & 37 & $\mathrm{x}$ & $\mathrm{x}$ & $\mathrm{x}$ & $\mathrm{x}$ & $\mathrm{x}$ & $\mathrm{x}$ \\
\hline Croton glandulosus L. & Subarbusto & 50 & $\mathrm{x}$ & $\mathrm{x}$ & $\mathrm{x}$ & $\mathrm{x}$ & $\mathrm{x}$ & $\mathrm{x}$ \\
\hline Croton goyazensis Müll. Arg. & Subarbusto & 79 & $\mathrm{x}$ & $\mathrm{x}$ & $\mathrm{x}$ & $\mathrm{x}$ & $\mathrm{x}$ & $\mathrm{x}$ \\
\hline Dalechampia caperonioides Baill. & Erva & 50 & $\mathrm{x}$ & $\mathrm{x}$ & $\mathrm{x}$ & $\mathrm{x}$ & $\mathrm{x}$ & $\mathrm{x}$ \\
\hline Maprounea brasiliensis A. St.-Hil. & Arbusto & 70 & $\mathrm{x}$ & $\mathrm{x}$ & $\mathrm{x}$ & $\mathrm{x}$ & $\mathrm{x}$ & $\mathrm{x}$ \\
\hline Sapium glandulatum (Vell.) Pax & Arbusto & 50 & $\mathrm{x}$ & $\mathrm{x}$ & $\mathrm{x}$ & & $\mathrm{x}$ & $\mathrm{x}$ \\
\hline \multicolumn{9}{|l|}{ FABACEAE (LEGUMINOSAE) } \\
\hline Acacia polyphylla DC. & Árvore & 96 & $\mathrm{x}$ & $\mathrm{x}$ & $\mathrm{x}$ & $\mathrm{x}$ & $\mathrm{x}$ & $\mathrm{x}$ \\
\hline Acosmium dasycarpum (Vogel) Yakovlev & Árvore & 50 & $\mathrm{x}$ & $\mathrm{x}$ & $\mathrm{x}$ & $\mathrm{x}$ & $\mathrm{x}$ & $\mathrm{x}$ \\
\hline Aeschynomene paniculata Willd. ex Vogel * & Subarbusto & 83 & $\mathrm{x}$ & $\mathrm{x}$ & $\mathrm{x}$ & $\mathrm{x}$ & $\mathrm{x}$ & $\mathrm{x}$ \\
\hline Aeschynomene paucifolia Vogel & Erva & 62 & $\mathrm{x}$ & & $\mathrm{x}$ & $\mathrm{x}$ & $\mathrm{x}$ & $\mathrm{x}$ \\
\hline Andira humilis Mart. ex Benth. & Subarbusto, arbusto & 70 & $\mathrm{x}$ & $\mathrm{x}$ & $\mathrm{x}$ & $\mathrm{x}$ & $\mathrm{x}$ & $\mathrm{x}$ \\
\hline Bauhinia dumosa Benth. & Subarbusto, arbusto & 29 & & $\mathrm{x}$ & $\mathrm{x}$ & $\mathrm{x}$ & & $\mathrm{x}$ \\
\hline Bauhinia holophylla (Bong.) Steud. & Subarbusto, arbusto & 83 & $\mathrm{x}$ & $\mathrm{x}$ & $\mathrm{x}$ & $\mathrm{x}$ & $\mathrm{x}$ & $\mathrm{x}$ \\
\hline Bauhinia rufa (Bong.) Steud. & Arbusto, árvore & 91 & $\mathrm{x}$ & $\mathrm{x}$ & $\mathrm{x}$ & $\mathrm{x}$ & $\mathrm{x}$ & $\mathrm{x}$ \\
\hline Bowdichia virgilioides Kunth & Árvore & 4 & & & & & & $\mathrm{x}$ \\
\hline Centrosema angustifolium (Kunth) Benth. & Trepadeira & 54 & $\mathrm{x}$ & $\mathrm{x}$ & $\mathrm{x}$ & $\mathrm{x}$ & $\mathrm{x}$ & $\mathrm{x}$ \\
\hline $\begin{array}{l}\text { Chamaecrista basifolia (Vogel) H. S. Irwin \& } \\
\text { Barbeby }\end{array}$ & Erva & 45 & $\mathrm{x}$ & $\mathrm{x}$ & $\mathrm{x}$ & $\mathrm{x}$ & $\mathrm{x}$ & $\mathrm{x}$ \\
\hline $\begin{array}{l}\text { Chamaecrista filicifolia (Benth.) H. S. Irwin \& } \\
\text { Barneby }\end{array}$ & Erva, subarbusto & 41 & $\mathrm{x}$ & $\mathrm{x}$ & $\mathrm{x}$ & $\mathrm{x}$ & $\mathrm{x}$ & $\mathrm{x}$ \\
\hline Chamaecrista flexuosa (L.) Greene * & Subarbusto & 54 & $\mathrm{x}$ & $\mathrm{x}$ & $\mathrm{x}$ & $\mathrm{x}$ & $\mathrm{x}$ & $\mathrm{x}$ \\
\hline Chamaecrista lundii (Benth.) H. S. Irwin \&Barneby & Erva & 16 & & & & $\mathrm{x}$ & $\mathrm{x}$ & $\mathrm{x}$ \\
\hline Chamacrista nictitans (L.) Moench & Erva, subarbusto & 87 & $\mathrm{x}$ & $\mathrm{x}$ & $\mathrm{x}$ & $\mathrm{x}$ & $\mathrm{x}$ & $\mathrm{x}$ \\
\hline $\begin{array}{l}\text { Chamaecrista planaltoana (Harms.) H. S. Irwin } \\
\text { \& Barneby }\end{array}$ & Erva & 8 & & & & $\mathrm{x}$ & & $\mathrm{x}$ \\
\hline Chamaecrista repens (Vogel) H. S. Irwin \& Barneby & Subarbusto & 20 & $\mathrm{x}$ & $\mathrm{x}$ & $\mathrm{x}$ & $\mathrm{x}$ & & $\mathrm{x}$ \\
\hline Chamaecrista serpens (L.) Greene & Erva & 37 & $\mathrm{x}$ & $\mathrm{x}$ & & $\mathrm{x}$ & $\mathrm{x}$ & $\mathrm{x}$ \\
\hline Clitoria guianensis (Aubl.) Benth. & Subarbusto & 96 & $\mathrm{x}$ & $\mathrm{x}$ & $\mathrm{x}$ & $\mathrm{x}$ & $\mathrm{x}$ & $\mathrm{x}$ \\
\hline Crotalaria flavicoma Benth. & Erva, subarbusto & 25 & $\mathrm{x}$ & $\mathrm{x}$ & & $\mathrm{x}$ & $\mathrm{x}$ & $\mathrm{x}$ \\
\hline Crotalaria unifoliolata Benth. & Subarbusto & 16 & $\mathrm{x}$ & & & $\mathrm{x}$ & $\mathrm{x}$ & \\
\hline Crotalaria velutina Benth. & Subarbusto & 12 & $\mathrm{x}$ & & & $\mathrm{x}$ & & $\mathrm{x}$ \\
\hline Dalbergia miscolobium Benth. & Árvore & 25 & & $\mathrm{x}$ & & $\mathrm{x}$ & & $\mathrm{x}$ \\
\hline Desmodium barbatum (L.) Benth. * & Subarbusto & 12 & & & $\mathrm{x}$ & & & $\mathrm{x}$ \\
\hline Desmodium incanum DC. * & Subarbusto & 70 & $\mathrm{x}$ & $\mathrm{x}$ & $\mathrm{x}$ & $\mathrm{x}$ & $\mathrm{x}$ & $\mathrm{x}$ \\
\hline Dimorphandra mollis Benth. & Árvore & 16 & & & $\mathrm{x}$ & $\mathrm{x}$ & & $\mathrm{x}$ \\
\hline Eriosema crinitum (Kunth) G. Don & Subarbusto & 96 & $\mathrm{x}$ & $\mathrm{x}$ & $\mathrm{x}$ & $\mathrm{x}$ & $\mathrm{x}$ & $\mathrm{x}$ \\
\hline
\end{tabular}


continuação

\begin{tabular}{|c|c|c|c|c|c|c|c|c|}
\hline Família/Espécie & Hábito & Freq $^{1}$ & $\mathrm{CM}$ & $\mathrm{CS}$ & FM & FS & MIM & MIS \\
\hline Eriosema defoliatum Benth & Erva, subarbusto & 33 & $\mathrm{x}$ & & $\mathrm{x}$ & & $\mathrm{x}$ & $\mathrm{x}$ \\
\hline Eriosema rufum (Kunth) G. Don & Erva & 8 & & $\mathrm{x}$ & & & $\mathrm{x}$ & \\
\hline Galactia decumbens (Benth.) Chodat \& Hassler & Erva & 79 & $\mathrm{x}$ & $\mathrm{x}$ & $\mathrm{x}$ & $\mathrm{x}$ & $\mathrm{x}$ & $\mathrm{x}$ \\
\hline Galactia grewiaefolia (Benth.) Taub. & Erva, subarbusto & 64 & $\mathrm{x}$ & $\mathrm{x}$ & $\mathrm{x}$ & $\mathrm{x}$ & $\mathrm{x}$ & $\mathrm{x}$ \\
\hline Mimosa albolanata Taub. & Arbusto, árvore & 87 & $\mathrm{x}$ & $\mathrm{x}$ & $\mathrm{x}$ & $\mathrm{x}$ & $\mathrm{x}$ & $\mathrm{x}$ \\
\hline Mimosa debilis Humb. \& Bonpl. ex Willd. & Subarbusto & 70 & & $\mathrm{x}$ & $\mathrm{x}$ & $\mathrm{x}$ & $\mathrm{x}$ & $\mathrm{x}$ \\
\hline Mimosa gracilis Benth. & Erva & 100 & $\mathrm{x}$ & $\mathrm{x}$ & $\mathrm{x}$ & $\mathrm{x}$ & $\mathrm{x}$ & $\mathrm{x}$ \\
\hline Mimosa lanuginosa Glaz. ex Burkart & Subarbusto & 62 & $\mathrm{x}$ & $\mathrm{x}$ & $\mathrm{x}$ & $\mathrm{x}$ & $\mathrm{x}$ & $\mathrm{x}$ \\
\hline Mimosa nuda Benth. & Arbusto & 83 & $\mathrm{x}$ & $\mathrm{x}$ & $\mathrm{x}$ & $\mathrm{x}$ & $\mathrm{x}$ & $\mathrm{x}$ \\
\hline Mimosa radula Benth. & Arbusto & 25 & $\mathrm{x}$ & $\mathrm{x}$ & $\mathrm{x}$ & $\mathrm{x}$ & & $\mathrm{x}$ \\
\hline Sclerolobium aureum (Tul.) Benth. & Árvore & 4 & & & & & & $\mathrm{x}$ \\
\hline $\begin{array}{l}\text { Sclerolobium paniculatum Vogel var. subvelutinum } \\
\text { Benth. }\end{array}$ & Árvore & 4 & & & & & & $\mathrm{x}$ \\
\hline Senna rugosa (G. Don) H. S. Irwin \& Barneby & Arbusto & 4 & $\mathrm{x}$ & & & & & \\
\hline Stryphnodendron adstringens (Mart.) Cov. & Árvore & 4 & & & & & & $\mathrm{x}$ \\
\hline Stylosanthes acuminata M. B. Ferr. \& Souza-Costa & Erva & 41 & $\mathrm{x}$ & & $\mathrm{x}$ & $\mathrm{x}$ & $\mathrm{x}$ & $\mathrm{x}$ \\
\hline $\begin{array}{l}\text { Stylosantes guianenesis (Aubl.) Sw. var. } \\
\text { microcephala M. B. Ferr. \& Souza-Costa }\end{array}$ & Erva & 45 & $\mathrm{x}$ & $\mathrm{x}$ & $\mathrm{x}$ & $\mathrm{x}$ & $\mathrm{x}$ & $\mathrm{x}$ \\
\hline $\begin{array}{l}\text { Stylosanthis guianenesis (Aubl.) Sw. var. pauciflora } \\
\text { M. B. Ferr. \& Souza-Costa }\end{array}$ & Erva & 33 & & & $\mathrm{x}$ & $\mathrm{x}$ & $\mathrm{x}$ & $\mathrm{x}$ \\
\hline Stylosanthes macrocephala M. B. Ferr. \& Souza-Costa & Erva & 50 & $\mathrm{x}$ & $\mathrm{x}$ & $\mathrm{x}$ & $\mathrm{x}$ & $\mathrm{x}$ & $\mathrm{x}$ \\
\hline Stylosanthes scabra Vogel & Subarbusto & 8 & & & $\mathrm{x}$ & & $\mathrm{x}$ & \\
\hline Tephrosia purpurea (L.) Pers. * & Erva & 25 & $\mathrm{x}$ & $\mathrm{x}$ & $\mathrm{x}$ & $\mathrm{x}$ & & $\mathrm{x}$ \\
\hline $\begin{array}{l}\text { Vigna linearis (Kunth) Maréchal, Mascherpa \& } \\
\text { Stainier (=Phaseolus linearis Kunth) }\end{array}$ & Trepadeira & 4 & & & & & $\mathrm{x}$ & \\
\hline $\begin{array}{l}\text { Vigna peduncularis (Kunth) Fawcett \& Rendle var. } \\
\text { clitoriodes (Mart. ex Benth.) Maréchal, Mascherpa } \\
\text { \& Stainier }\end{array}$ & Trepadeira & 4 & & & & & & $\mathrm{x}$ \\
\hline Zornia latifolia $\mathrm{Sm}$. & Erva & 62 & & $\mathrm{x}$ & $\mathrm{x}$ & $\mathrm{x}$ & $\mathrm{x}$ & $\mathrm{x}$ \\
\hline Zornia vestita Mohlenbr. & Erva & 29 & $\mathrm{x}$ & $\mathrm{x}$ & $\mathrm{x}$ & $\mathrm{x}$ & $\mathrm{x}$ & \\
\hline \multicolumn{9}{|l|}{ GENTIANACEAE } \\
\hline Deianira chiquitana Herzog & Subarbusto & 4 & & & & $\mathrm{x}$ & & \\
\hline $\begin{array}{l}\text { Irlbachia alata (Aubl.) Maas subsp. alata } \\
\text { GESNERIACEAE }\end{array}$ & Subarbusto & 25 & & & & $\mathrm{x}$ & $\mathrm{x}$ & $\mathrm{x}$ \\
\hline Sinningia allagophylla (Mart.) Wiehler & Erva & 37 & $\mathrm{x}$ & $\mathrm{x}$ & $\mathrm{x}$ & $\mathrm{x}$ & $\mathrm{x}$ & $\mathrm{x}$ \\
\hline HYPOXIDACEAE & & & & & & & & \\
\hline $\begin{array}{l}\text { Curculigo scorzonerifolia (Lam.) Baker } \\
\text { IRIDACEAE }\end{array}$ & Erva & 16 & & $\mathrm{x}$ & $\mathrm{x}$ & & $\mathrm{x}$ & \\
\hline Alophia cf. sellowiana Klatt & Erva & 4 & & & & & & $\mathrm{x}$ \\
\hline Cipura paludosa Aubl. & Erva & 16 & $\mathrm{x}$ & & & $\mathrm{x}$ & $\mathrm{x}$ & $\mathrm{x}$ \\
\hline Sisyrinchium restioides Spreng. & Erva & 29 & $\mathrm{x}$ & $\mathrm{x}$ & $\mathrm{x}$ & $\mathrm{x}$ & $\mathrm{x}$ & $\mathrm{x}$ \\
\hline Trimezia sp. (CRM 690) & Erva & 70 & & $\mathrm{x}$ & $\mathrm{x}$ & $\mathrm{x}$ & & $\mathrm{x}$ \\
\hline \multicolumn{9}{|l|}{ LAMIACEAE } \\
\hline Aegiphila verticillata Vell & Árvore & 54 & $\mathrm{x}$ & & $\mathrm{x}$ & $\mathrm{x}$ & $\mathrm{x}$ & $\mathrm{x}$ \\
\hline Eriope crassipes Benth. & Erva & 4 & & & & & $\mathrm{x}$ & \\
\hline Hypenia densiflora (Pohl ex Benth.) Harley & Subarbusto, arbusto & 45 & $\mathrm{x}$ & $\mathrm{x}$ & $\mathrm{x}$ & $\mathrm{x}$ & $\mathrm{x}$ & $\mathrm{x}$ \\
\hline Hypenia macrantha (A. St.-Hil. ex Benth.) Harley & Subarbusto & 29 & $\mathrm{x}$ & $\mathrm{x}$ & & $\mathrm{x}$ & $\mathrm{x}$ & $\mathrm{x}$ \\
\hline Hyptis crinita Benth. & Arbusto & 75 & $\mathrm{x}$ & $\mathrm{x}$ & $\mathrm{x}$ & $\mathrm{x}$ & $\mathrm{x}$ & $\mathrm{x}$ \\
\hline Hyptis desortorum Pohl ex Benth. & Arbusto & 4 & & & & $\mathrm{x}$ & & \\
\hline Hyptis glomerata Mart. ex Schrank & Arbusto & 4 & & & & $\mathrm{x}$ & & \\
\hline Hyptis lutescens Pohl ex Benth. & Arbusto & 12 & & & & $\mathrm{x}$ & & $\mathrm{x}$ \\
\hline Hyptis lythroides Pohl ex Benth. & Subarbusto & 4 & & & & & & $\mathrm{x}$ \\
\hline Hyptis nudicaulis Benth. & Erva & 4 & & & & $\mathrm{x}$ & & \\
\hline Hyptis suaveolens (L.) Poit. * & Erva & 45 & $\mathrm{x}$ & $\mathrm{x}$ & $\mathrm{x}$ & & $\mathrm{x}$ & $\mathrm{x}$ \\
\hline
\end{tabular}


continuação

\begin{tabular}{|c|c|c|c|c|c|c|c|c|}
\hline Família/Espécie & Hábito & Freq $^{1}$ & $\mathrm{CM}$ & $\mathrm{CS}$ & FM & FS & MIM & MIS \\
\hline Hyptis tenuifolia Epling & Subarbusto & 96 & $\mathrm{x}$ & $\mathrm{x}$ & $\mathrm{x}$ & $\mathrm{x}$ & $\mathrm{x}$ & $\mathrm{x}$ \\
\hline Hyptis villosa Pohl ex Benth. & Erva & 91 & $\mathrm{x}$ & $\mathrm{x}$ & $\mathrm{x}$ & $\mathrm{x}$ & $\mathrm{x}$ & $\mathrm{x}$ \\
\hline Hyptis sp. (CRM 799) & Subarbusto & 4 & & & & & & $\mathrm{x}$ \\
\hline Marsypianthes montana Benth. & Subarbusto & 8 & & & $\mathrm{x}$ & & $\mathrm{x}$ & \\
\hline Salvia brevipes Benth. & Erva & 41 & $\mathrm{x}$ & $\mathrm{x}$ & $\mathrm{x}$ & $\mathrm{x}$ & $\mathrm{x}$ & $\mathrm{x}$ \\
\hline \multicolumn{9}{|l|}{ LORANTHACEAE } \\
\hline Phthirusa ovata (DC.) Eichler & Hemiparasita & 33 & $\mathrm{x}$ & $\mathrm{x}$ & & & & \\
\hline Struthanthus flexicaulis (Mart.) Mart. & Hemiparasita & 37 & $\mathrm{x}$ & $\mathrm{x}$ & & $\mathrm{x}$ & & \\
\hline \multicolumn{9}{|l|}{ LYTHRACEAE } \\
\hline Cuphea ferruginea Pohl ex Koehne & Erva, subarbusto & 64 & $\mathrm{x}$ & $\mathrm{x}$ & $\mathrm{x}$ & $\mathrm{x}$ & $\mathrm{x}$ & $\mathrm{x}$ \\
\hline Cuphea linarioides Cham. \& Schltdl. & Erva, subarbusto & 96 & $\mathrm{x}$ & $\mathrm{x}$ & $\mathrm{x}$ & $\mathrm{x}$ & $\mathrm{x}$ & $\mathrm{x}$ \\
\hline Cuphea micrantha Kunth & Erva, subarbusto & 8 & & & $\mathrm{x}$ & & $\mathrm{x}$ & \\
\hline Cuphea spermacoce A. St.-Hil. & Erva, subarbusto & 87 & $\mathrm{x}$ & $\mathrm{x}$ & $\mathrm{x}$ & $\mathrm{x}$ & $\mathrm{x}$ & $\mathrm{x}$ \\
\hline Diplusodon villosus Pohl & Subarbusto & 75 & $\mathrm{x}$ & $\mathrm{x}$ & $\mathrm{x}$ & $\mathrm{x}$ & $\mathrm{x}$ & $\mathrm{x}$ \\
\hline Diplusodon virgatus Pohl & Arbusto, Árvore & 12 & & $\mathrm{x}$ & $\mathrm{x}$ & & & \\
\hline \multicolumn{9}{|l|}{ MALPIGHIACEAE } \\
\hline Banisteriopsis argyrophylla (A. Juss.) B. Gates & $\begin{array}{l}\text { Trepadeira, } \\
\text { subarbusto }\end{array}$ & 4 & & $\mathrm{x}$ & & & & \\
\hline Banisteriopsis campestris (A. Juss.) E. Little & Subarbusto & 87 & $\mathrm{x}$ & $\mathrm{x}$ & $\mathrm{x}$ & $\mathrm{x}$ & $\mathrm{x}$ & $\mathrm{x}$ \\
\hline Banisteriopsis laevifolia (A. Juss.) B. Gates & Arbusto, árvore & 8 & & & $\mathrm{x}$ & & & $\mathrm{x}$ \\
\hline Banisteriopsis latifolia (A. Juss.) B. Gates & Arbusto & 4 & $\mathrm{x}$ & & & & & \\
\hline Banisteriopsis malifolia (Nees \& Mart.) B. Gates & Arbusto, árvore & 4 & & & & & & $\mathrm{x}$ \\
\hline Banisteriopsis stellaris (Griseb.) B. Gates & $\begin{array}{l}\text { Trepadeira, } \\
\text { subarbusto }\end{array}$ & 4 & & $\mathrm{x}$ & & & & \\
\hline Byrsonima basiloba A. Juss. & Arbusto & 12 & $\mathrm{x}$ & & & $\mathrm{x}$ & $\mathrm{x}$ & \\
\hline Byrsonima coccolobifolia Kunth & Árvore & 12 & $\mathrm{x}$ & $\mathrm{x}$ & & & $\mathrm{x}$ & \\
\hline Byrsonima rigida A. Juss. & Subarbusto, arbusto & 29 & $\mathrm{x}$ & $\mathrm{x}$ & & $\mathrm{x}$ & $\mathrm{x}$ & $\mathrm{x}$ \\
\hline Byrsonima subterranea Brade \& Markgr. & Subarbusto, arbusto & 4 & & $\mathrm{x}$ & & & & \\
\hline Byrsonima verbascifolia (L.) L. C. Rich. ex A. Juss. & Arbusto, árvore & 25 & & $\mathrm{x}$ & & $\mathrm{x}$ & $\mathrm{x}$ & $\mathrm{x}$ \\
\hline Byrsonima sp. (CM 607) & Arbusto & 4 & $\mathrm{x}$ & & & & & \\
\hline Camarea affinis A. St.-Hil. & Erva & 25 & $\mathrm{x}$ & $\mathrm{x}$ & & $\mathrm{x}$ & & $\mathrm{x}$ \\
\hline Heteropterys eglandulosa A. Juss. & Arbusto & 4 & $\mathrm{x}$ & & & & & \\
\hline Peixotoa goiana C. E. Anderson & Subarbusto & 62 & $\mathrm{x}$ & $\mathrm{x}$ & $\mathrm{x}$ & $\mathrm{x}$ & $\mathrm{x}$ & $\mathrm{x}$ \\
\hline Peixotoa reticulata Griseb. & Arbusto & 4 & & & $\mathrm{x}$ & & & \\
\hline Heteroptelys campestris A. Juss. & Subarbusto, arbusto & 29 & & $\mathrm{x}$ & $\mathrm{x}$ & & & $\mathrm{x}$ \\
\hline Peixotoa tomentosa A. Juss. & Subarbusto & 4 & $\mathrm{x}$ & & & & & \\
\hline Pterandra pyroidea A. Juss. & Arbusto & 12 & $\mathrm{x}$ & & $\mathrm{x}$ & & & $\mathrm{x}$ \\
\hline Tetrapterys ambigua (A. Juss.) Nied. & Subarbusto, arbusto & 50 & $\mathrm{x}$ & $\mathrm{x}$ & $\mathrm{x}$ & & $\mathrm{x}$ & $\mathrm{x}$ \\
\hline \multicolumn{9}{|l|}{ MALVACEAE } \\
\hline Byttneria scalpellata Pohl & Erva & 96 & $\mathrm{x}$ & $\mathrm{x}$ & $\mathrm{x}$ & $\mathrm{x}$ & $\mathrm{x}$ & $\mathrm{x}$ \\
\hline Eriotheca pubescens (Mart. \& Zucc.) Schott \& Endl. & Árvore & 8 & $\mathrm{x}$ & & & & & $\mathrm{x}$ \\
\hline Peltaea lasiantha Krapov. \& Cristóbal & Erva, subarbusto & 50 & & & $\mathrm{x}$ & & $\mathrm{x}$ & \\
\hline Peltaea riedellii (Gürke) Standl. & Subarbusto & 8 & & & $\mathrm{x}$ & & $\mathrm{x}$ & \\
\hline Peltaea sp. (CRM 598) & Subarbusto & 4 & & & $\mathrm{x}$ & & & \\
\hline Sida cerradoensis Krapov. & Subarbusto & 33 & $\mathrm{x}$ & $\mathrm{x}$ & $\mathrm{x}$ & $\mathrm{x}$ & & $\mathrm{x}$ \\
\hline Sida cordifolia L. * & Arbusto & 50 & $\mathrm{x}$ & $\mathrm{x}$ & $\mathrm{x}$ & $\mathrm{x}$ & $\mathrm{x}$ & $\mathrm{x}$ \\
\hline Sida linifolia Cav. * & Erva & 91 & $\mathrm{x}$ & $\mathrm{x}$ & $\mathrm{x}$ & $\mathrm{x}$ & $\mathrm{x}$ & $\mathrm{x}$ \\
\hline Sida rhombifolia L. * & Subarbusto & 45 & $\mathrm{x}$ & $\mathrm{x}$ & $\mathrm{x}$ & & $\mathrm{x}$ & $\mathrm{x}$ \\
\hline Sida viarum A. St.-Hil. & Subarbusto & 66 & $\mathrm{x}$ & $\mathrm{x}$ & $\mathrm{x}$ & $\mathrm{x}$ & $\mathrm{x}$ & $\mathrm{x}$ \\
\hline Triumffeta semitriloba Jacq. * & Subarbusto, arbusto & 37 & $\mathrm{x}$ & $\mathrm{x}$ & & $\mathrm{x}$ & & $\mathrm{x}$ \\
\hline Waltheria communis A. St.-Hil. & Erva & 20 & & & $\mathrm{x}$ & $\mathrm{x}$ & $\mathrm{x}$ & $\mathrm{x}$ \\
\hline Waltheria indica $\mathrm{L}$. & Arbusto & 41 & $\mathrm{x}$ & $\mathrm{x}$ & $\mathrm{x}$ & $\mathrm{x}$ & $\mathrm{x}$ & $\mathrm{x}$ \\
\hline \multicolumn{9}{|l|}{ MELASTOMATACEAE } \\
\hline Cambessedesia espora DC. & Erva, subarbusto & 41 & $\mathrm{x}$ & $\mathrm{x}$ & $\mathrm{x}$ & $\mathrm{x}$ & $\mathrm{x}$ & $\mathrm{x}$ \\
\hline
\end{tabular}


continuação

\begin{tabular}{|c|c|c|c|c|c|c|c|c|}
\hline Família/Espécie & Hábito & Freq $^{1}$ & $\mathrm{CM}$ & $\mathrm{CS}$ & FM & FS & MIM & MIS \\
\hline Miconia albicans (Sw.) Triana & Arbusto, árvore & 100 & $\mathrm{x}$ & $\mathrm{x}$ & $\mathrm{x}$ & $\mathrm{x}$ & $\mathrm{x}$ & $\mathrm{x}$ \\
\hline Miconia fallax A. DC. & Arbusto, árvore & 37 & $\mathrm{x}$ & & $\mathrm{x}$ & & $\mathrm{x}$ & $\mathrm{x}$ \\
\hline Pterolepis perpusilla (Naudin) Cogn. & Erva & 45 & $\mathrm{x}$ & & & $\mathrm{x}$ & $\mathrm{x}$ & $\mathrm{x}$ \\
\hline Pterolepis repanda (DC.) Triana & Erva, subarbusto & 37 & $\mathrm{x}$ & $\mathrm{x}$ & $\mathrm{x}$ & $\mathrm{x}$ & $\mathrm{x}$ & $\mathrm{x}$ \\
\hline Tibouchina aegopogon (Naudin) Cogn. & Subarbusto, arbusto & 12 & $\mathrm{x}$ & & & & $\mathrm{x}$ & \\
\hline $\begin{array}{l}\text { Tibouchina stenocarpa (Schrank \& Mart. ex DC.) } \\
\text { Cogn. }\end{array}$ & Árvore & 41 & $\mathrm{x}$ & $\mathrm{x}$ & $\mathrm{x}$ & $\mathrm{x}$ & $\mathrm{x}$ & $\mathrm{x}$ \\
\hline \multicolumn{9}{|l|}{ MENISPERMACEAE } \\
\hline $\begin{array}{l}\text { Cissampelos ovalifolia DC. } \\
\text { MORACEAE }\end{array}$ & \multicolumn{7}{|c|}{ MORACEAE } & $\mathrm{x}$ \\
\hline Brosimum gaudichaudii Trécul & Arbusto & 20 & $\mathrm{x}$ & & & $\mathrm{x}$ & $\mathrm{x}$ & $\mathrm{x}$ \\
\hline \multicolumn{9}{|l|}{ MYRSINACEAE } \\
\hline $\begin{array}{l}\text { Myrsine guianensis (Aubl.) Kuntze } \\
\text { MYRTACEAE }\end{array}$ & \multicolumn{5}{|c|}{ MYRTACEAE } & $\mathrm{x}$ & $\mathrm{x}$ & $\mathrm{x}$ \\
\hline Campomanesia adamantium (Cambess.) O. Berg & Subarbusto, arbusto & 4 & $\mathrm{x}$ & & & & & \\
\hline Campomanesia pubescens (A. DC.) O. Berg & Arbusto & 100 & $\mathrm{x}$ & $\mathrm{x}$ & $\mathrm{x}$ & $\mathrm{x}$ & $\mathrm{x}$ & $\mathrm{x}$ \\
\hline Campomanesia xanthocarpa O. Berg & Árvore & 91 & $\mathrm{x}$ & $\mathrm{x}$ & $\mathrm{x}$ & $\mathrm{x}$ & $\mathrm{x}$ & $\mathrm{x}$ \\
\hline Eugenia bracteata Vell. & Arbusto, árvore & 91 & $\mathrm{x}$ & $\mathrm{x}$ & $\mathrm{x}$ & $\mathrm{x}$ & $\mathrm{x}$ & $\mathrm{x}$ \\
\hline Eugenia complicata $\mathrm{O}$. Berg & Arbusto & 33 & & $\mathrm{x}$ & $\mathrm{x}$ & & $\mathrm{x}$ & $\mathrm{x}$ \\
\hline Myrcia cordiifolia $\mathrm{O}$. Berg & Arbusto & 33 & $\mathrm{x}$ & $\mathrm{x}$ & & $\mathrm{x}$ & $\mathrm{x}$ & $\mathrm{x}$ \\
\hline Myrcia decrescens $\mathrm{O}$. Berg & Erva & 83 & $\mathrm{x}$ & $\mathrm{x}$ & $\mathrm{x}$ & $\mathrm{x}$ & $\mathrm{x}$ & $\mathrm{x}$ \\
\hline Myrcia hiemalis Cambess. & Subarbusto & 96 & $\mathrm{x}$ & $\mathrm{x}$ & $\mathrm{x}$ & $\mathrm{x}$ & $\mathrm{x}$ & $\mathrm{x}$ \\
\hline Myrcia linearifolia Cambess. & Erva & 12 & $\mathrm{x}$ & $\mathrm{x}$ & & $\mathrm{x}$ & & \\
\hline Myrcia rhodosepala Kiaerk. & Subarbusto & 4 & & & & & $\mathrm{x}$ & \\
\hline Myrcia rostrata DC. & Arbusto, árvore & 4 & $\mathrm{x}$ & & & & & \\
\hline Myrcia tomentosa (Aubl.) DC. & Árvore & 25 & & $\mathrm{x}$ & $\mathrm{x}$ & $\mathrm{x}$ & & $\mathrm{x}$ \\
\hline Psidium australe Cambess. & Arbusto & 70 & $\mathrm{x}$ & $\mathrm{x}$ & $\mathrm{x}$ & $\mathrm{x}$ & $\mathrm{x}$ & $\mathrm{x}$ \\
\hline Psidium cinereum Mart. ex DC. & Arbusto & 33 & $\mathrm{x}$ & $\mathrm{x}$ & $\mathrm{x}$ & $\mathrm{x}$ & $\mathrm{x}$ & $\mathrm{x}$ \\
\hline Psidium firmum $\mathrm{O}$. Berg & Arbusto & 96 & $\mathrm{x}$ & $\mathrm{x}$ & $\mathrm{x}$ & $\mathrm{x}$ & $\mathrm{x}$ & $\mathrm{x}$ \\
\hline Psidium laruotteanum Cambess. & Arbusto & 29 & & & $\mathrm{x}$ & & & $\mathrm{x}$ \\
\hline $\begin{array}{l}\text { Psidium salutare (Kunth) O. Berg var. mucronatum } \\
\text { (Cambess.) Landrum }\end{array}$ & Subarbusto & 25 & & & & $\mathrm{x}$ & $\mathrm{x}$ & $\mathrm{x}$ \\
\hline $\begin{array}{l}\text { Psidium salutare (Kunth) O. Berg var. pohlianum } \\
\text { (O.Berg) Landrum }\end{array}$ & Arbusto, árvore & 20 & $\mathrm{x}$ & $\mathrm{x}$ & $\mathrm{x}$ & & & $\mathrm{x}$ \\
\hline Psidium sp. (CM 180) & Subarbusto & 41 & $\mathrm{x}$ & $\mathrm{x}$ & & $\mathrm{x}$ & $\mathrm{x}$ & $\mathrm{x}$ \\
\hline \multicolumn{9}{|l|}{ OCHNACEAE } \\
\hline Ouratea floribunda (A. St.-Hil) Engl. & Subarbusto, arbusto & 54 & $\mathrm{x}$ & $\mathrm{x}$ & $\mathrm{x}$ & $\mathrm{x}$ & $\mathrm{x}$ & $\mathrm{x}$ \\
\hline Ouratea nervosa (A. St.-Hil.) Engl. & Arbusto & 41 & $\mathrm{x}$ & $\mathrm{x}$ & & $\mathrm{x}$ & $\mathrm{x}$ & $\mathrm{x}$ \\
\hline \multicolumn{9}{|l|}{ ORCHIDACEAE } \\
\hline Epistephium sclerophyllum Lindl. & Erva terrestre & 4 & & & & $\mathrm{x}$ & & \\
\hline Habenaria hexaptera Lindl. & Erva terrestre & 16 & $\mathrm{x}$ & $\mathrm{x}$ & $\mathrm{x}$ & & & $\mathrm{x}$ \\
\hline Habenaria obtusa Lindl. & Erva terrestre & 20 & $\mathrm{x}$ & & & & & $\mathrm{x}$ \\
\hline \multicolumn{9}{|l|}{ OROBANCHACEAE } \\
\hline Buchnera rosea Kunth & Subarbusto & 29 & $\mathrm{x}$ & $\mathrm{x}$ & $\mathrm{x}$ & $\mathrm{x}$ & $\mathrm{x}$ & $\mathrm{x}$ \\
\hline Esterhazya splendida J. C. Mikan & Arbusto & 4 & $\mathrm{x}$ & & & & & \\
\hline \multicolumn{9}{|l|}{ OXALIDACEAE } \\
\hline Oxalis densifolia Mart. \& Zucc. ex Zucc. & Erva, subarbusto & 100 & $\mathrm{x}$ & $\mathrm{x}$ & $\mathrm{x}$ & $\mathrm{x}$ & $\mathrm{x}$ & $\mathrm{x}$ \\
\hline Oxalis suborbiculata Lourteig & Erva, subarbusto & 96 & $\mathrm{x}$ & $\mathrm{x}$ & $\mathrm{x}$ & $\mathrm{x}$ & $\mathrm{x}$ & $\mathrm{x}$ \\
\hline \multicolumn{9}{|l|}{ PASSIFLORACEAE } \\
\hline Passiflora clathrata Mast. & Trepadeira, erva & 58 & $\mathrm{x}$ & $\mathrm{x}$ & $\mathrm{x}$ & $\mathrm{x}$ & $\mathrm{x}$ & $\mathrm{x}$ \\
\hline \multicolumn{9}{|l|}{ POACEAE } \\
\hline Agenium leptocladum (Hack.) Clayton & Erva & 83 & $\mathrm{x}$ & $\mathrm{x}$ & $\mathrm{x}$ & $\mathrm{x}$ & $\mathrm{x}$ & $\mathrm{x}$ \\
\hline Andropogon bicornis L. * & Erva & 8 & & $\mathrm{x}$ & & & & $\mathrm{x}$ \\
\hline Andropogon fastigiatus Sw. * & Erva & 8 & & $\mathrm{x}$ & $\mathrm{x}$ & & & \\
\hline
\end{tabular}


continuação

\begin{tabular}{|c|c|c|c|c|c|c|c|c|}
\hline Família/Espécie & Hábito & Freq $^{1}$ & $\mathrm{CM}$ & $\mathrm{CS}$ & FM & FS & MIM & MIS \\
\hline Andropogon gayanus Kunth * & Erva & 58 & $\mathrm{x}$ & $\mathrm{x}$ & $\mathrm{x}$ & $\mathrm{x}$ & $\mathrm{x}$ & $\mathrm{x}$ \\
\hline Andropogon leucostachyus Kunth * & Erva & 54 & $\mathrm{x}$ & $\mathrm{x}$ & $\mathrm{x}$ & $\mathrm{x}$ & $\mathrm{x}$ & $\mathrm{x}$ \\
\hline Anthaenantia lanata (Kunth) Benth. & Erva & 70 & $\mathrm{x}$ & $\mathrm{x}$ & $\mathrm{x}$ & $\mathrm{x}$ & $\mathrm{x}$ & $\mathrm{x}$ \\
\hline Aristida gibbosa (Nees) Kunth & Erva & 16 & & $\mathrm{x}$ & $\mathrm{x}$ & $\mathrm{x}$ & & $\mathrm{x}$ \\
\hline Aristida recurvata Kunth & Erva & 4 & & & & & & \\
\hline Aristida setifolia Kunth & Erva & 45 & $\mathrm{x}$ & & $\mathrm{x}$ & $\mathrm{x}$ & $\mathrm{x}$ & $\mathrm{x}$ \\
\hline Aristida torta (Nees) Kunth & Erva & 29 & & & $\mathrm{x}$ & $\mathrm{x}$ & $\mathrm{x}$ & $\mathrm{x}$ \\
\hline Axonopus canescens (Nees ex Trin.) Pilg. & Erva & 4 & & & & $\mathrm{x}$ & & \\
\hline Axonopus marginatus (Trin.) Chase & Erva & 70 & $\mathrm{x}$ & $\mathrm{x}$ & $\mathrm{x}$ & $\mathrm{x}$ & $\mathrm{x}$ & $\mathrm{x}$ \\
\hline Digitaria filiformes (L.) Koeler & Erva & 29 & & $\mathrm{x}$ & $\mathrm{x}$ & $\mathrm{x}$ & $\mathrm{x}$ & $\mathrm{x}$ \\
\hline Digitaria neesiana Henrard & Erva & 4 & & & & $\mathrm{x}$ & & \\
\hline Echinolaena inflexa (Poir.) Chase & Erva & 100 & $\mathrm{x}$ & $\mathrm{x}$ & $\mathrm{x}$ & $\mathrm{x}$ & $\mathrm{x}$ & $\mathrm{x}$ \\
\hline Elionurus muticus (Spreng.) Kuntze & Erva & 58 & & & $\mathrm{x}$ & $\mathrm{x}$ & $\mathrm{x}$ & $\mathrm{x}$ \\
\hline Eragrostis polytricha Nees & Erva & 70 & $\mathrm{x}$ & $\mathrm{x}$ & $\mathrm{x}$ & $\mathrm{x}$ & $\mathrm{x}$ & $\mathrm{x}$ \\
\hline Eragrostis rufescens Schrad. ex Schult. & Erva & 20 & $\mathrm{x}$ & $\mathrm{x}$ & & $\mathrm{x}$ & & $\mathrm{x}$ \\
\hline Gymnopogon doellii Boechat \& Valls & Erva & 16 & & $\mathrm{x}$ & & $\mathrm{x}$ & & $\mathrm{x}$ \\
\hline Gymmonpogon foliosum (Willd.) Nees & Erva & 20 & & & $\mathrm{x}$ & & $\mathrm{x}$ & $\mathrm{x}$ \\
\hline $\begin{array}{l}\text { Hyparrhenia bracteata (Humb. \& Bonpl. ex Willd.) } \\
\text { Stapf }\end{array}$ & Erva & 37 & $\mathrm{x}$ & $\mathrm{x}$ & $\mathrm{x}$ & $\mathrm{x}$ & $\mathrm{x}$ & $\mathrm{x}$ \\
\hline Hyparrhenia rufa (Nees) Stapf* & Erva & 45 & $\mathrm{x}$ & $\mathrm{x}$ & $\mathrm{x}$ & $\mathrm{x}$ & $\mathrm{x}$ & $\mathrm{x}$ \\
\hline Ichnanthus camporum Swallen & Erva & 100 & $\mathrm{x}$ & $\mathrm{x}$ & $\mathrm{x}$ & $\mathrm{x}$ & $\mathrm{x}$ & $\mathrm{x}$ \\
\hline $\begin{array}{l}\text { Melinis minutiflora } \text { P. Beauv. (cultivar Cabelo-de- } \\
\text { Negro) * }\end{array}$ & Erva & 100 & $\mathrm{x}$ & $\mathrm{x}$ & $\mathrm{x}$ & $\mathrm{x}$ & $\mathrm{x}$ & $\mathrm{x}$ \\
\hline Melinis minutiflora P. Beauv (cultivar Roxo) * & Erva & 100 & $\mathrm{x}$ & $\mathrm{x}$ & $\mathrm{x}$ & $\mathrm{x}$ & $\mathrm{x}$ & $\mathrm{x}$ \\
\hline Melinis repens (Willd.) Zizka* & Erva & 4 & $\mathrm{x}$ & $\mathrm{x}$ & $\mathrm{x}$ & $\mathrm{x}$ & $\mathrm{x}$ & $\mathrm{x}$ \\
\hline Panicum campestre Nees ex Trin. & Erva & 8 & & $\mathrm{x}$ & & & & $\mathrm{x}$ \\
\hline Panicum olyroides Kunth var. hirsutum Henrard & Erva & 33 & $\mathrm{x}$ & $\mathrm{x}$ & $\mathrm{x}$ & $\mathrm{x}$ & $\mathrm{x}$ & $\mathrm{x}$ \\
\hline Panicum olyroides Kunth var. olyroides & Erva & 87 & $\mathrm{x}$ & $\mathrm{x}$ & & $\mathrm{x}$ & $\mathrm{x}$ & $\mathrm{x}$ \\
\hline Panicum peladoense Henrard & Erva & 33 & $\mathrm{x}$ & $\mathrm{x}$ & $\mathrm{x}$ & & & $\mathrm{x}$ \\
\hline Paspalum ammodes Trin. & Erva & 25 & & & $\mathrm{x}$ & & $\mathrm{x}$ & \\
\hline Paspalum burchellii Munro ex Oliv. & Erva & 12 & $\mathrm{x}$ & & $\mathrm{x}$ & $\mathrm{x}$ & & \\
\hline Paspalum clavuliferum $\mathrm{C}$. Wright & Erva & 16 & & $\mathrm{x}$ & & $\mathrm{x}$ & $\mathrm{x}$ & $\mathrm{x}$ \\
\hline Paspalum erianthum Nees ex Trin. & Erva & 91 & $\mathrm{x}$ & $\mathrm{x}$ & $\mathrm{x}$ & $\mathrm{x}$ & $\mathrm{x}$ & $\mathrm{x}$ \\
\hline Paspalum еисотит Nees ex Trin. & Erva & 12 & & $\mathrm{x}$ & & $\mathrm{x}$ & & $\mathrm{x}$ \\
\hline Paspalum gardnerianum Nees & Erva & 79 & $\mathrm{x}$ & $\mathrm{x}$ & $\mathrm{x}$ & $\mathrm{x}$ & $\mathrm{x}$ & $\mathrm{x}$ \\
\hline Paspalum geminiflorum Steud. & Erva & 54 & & $\mathrm{x}$ & $\mathrm{x}$ & $\mathrm{x}$ & $\mathrm{x}$ & $\mathrm{x}$ \\
\hline $\begin{array}{l}\text { Paspalum guenoarum Arechav. var. rojasii (Hack.) } \\
\text { Parodi ex Burkart }\end{array}$ & Erva & 75 & $\mathrm{x}$ & $\mathrm{x}$ & $\mathrm{x}$ & $\mathrm{x}$ & $\mathrm{x}$ & $\mathrm{x}$ \\
\hline Paspalum paniculatum L. * & Erva & 37 & & $\mathrm{x}$ & $\mathrm{x}$ & $\mathrm{x}$ & $\mathrm{x}$ & $\mathrm{x}$ \\
\hline Paspalum pectinatum Nees ex Trin. & Erva & 70 & $\mathrm{x}$ & $\mathrm{x}$ & $\mathrm{x}$ & $\mathrm{x}$ & $\mathrm{x}$ & $\mathrm{x}$ \\
\hline Paspalum pilosum Lam. & Erva & 58 & $\mathrm{x}$ & $\mathrm{x}$ & $\mathrm{x}$ & $\mathrm{x}$ & $\mathrm{x}$ & $\mathrm{x}$ \\
\hline Paspalum plicatulum Michx. * & Erva & 8 & & $\mathrm{x}$ & & & & $\mathrm{x}$ \\
\hline Paspalum polyphyllum Nees ex Trin. & Erva & 25 & & $\mathrm{x}$ & $\mathrm{x}$ & & $\mathrm{x}$ & $\mathrm{x}$ \\
\hline Paspalum stellatum Humb. \& Bonpl. ex Flüggé & Erva & 62 & $\mathrm{x}$ & $\mathrm{x}$ & $\mathrm{x}$ & $\mathrm{x}$ & $\mathrm{x}$ & $\mathrm{x}$ \\
\hline Paspalum trichostomum Hack. & Erva & 12 & $\mathrm{x}$ & $\mathrm{x}$ & $\mathrm{x}$ & $\mathrm{x}$ & $\mathrm{x}$ & $\mathrm{x}$ \\
\hline Pennisetum pedicelatum Trin. * & Erva & 58 & & $\mathrm{x}$ & & & & \\
\hline $\begin{array}{l}\text { Pennisetum polystachion (L.) Schult. (=Pennisetum } \\
\text { setosum (Sw.) L. Rich.) * }\end{array}$ & Erva & 70 & $\mathrm{x}$ & & $\mathrm{x}$ & $\mathrm{x}$ & $\mathrm{x}$ & $\mathrm{x}$ \\
\hline $\begin{array}{l}\text { Schizachyrium microstachyum (Desv. ex Ham.) } \\
\text { Roseng. }\end{array}$ & Erva & 100 & $\mathrm{x}$ & $\mathrm{x}$ & $\mathrm{x}$ & $\mathrm{x}$ & $\mathrm{x}$ & $\mathrm{x}$ \\
\hline Schizachyrium sanguineum (Retz.) Alston * & Erva & 29 & & $\mathrm{x}$ & $\mathrm{x}$ & $\mathrm{x}$ & & $\mathrm{x}$ \\
\hline $\begin{array}{l}\text { Schizachyrium sanguineum (Retz.) Alston subvar. } \\
\text { semiberbe (Nees) Roberty * }\end{array}$ & Erva & 29 & $\mathrm{x}$ & $\mathrm{x}$ & $\mathrm{x}$ & $\mathrm{x}$ & $\mathrm{x}$ & \\
\hline Schizachyrium tenerum Nees & Erva & 100 & $\mathrm{x}$ & $\mathrm{x}$ & $\mathrm{x}$ & $\mathrm{x}$ & $\mathrm{x}$ & $\mathrm{x}$ \\
\hline
\end{tabular}


continuação

\begin{tabular}{|c|c|c|c|c|c|c|c|c|}
\hline Família/Espécie & Hábito & Freq $^{1}$ & $\mathrm{CM}$ & CS & FM & FS & MIM & MIS \\
\hline Setaria parviflora (Poir.) Kerguélen & Erva & 64 & $\mathrm{x}$ & $\mathrm{x}$ & $\mathrm{x}$ & $\mathrm{x}$ & $\mathrm{x}$ & $\mathrm{x}$ \\
\hline Sorghastrum minarum (Nees) Hitchc. & Erva & 29 & $\mathrm{x}$ & $\mathrm{x}$ & $\mathrm{x}$ & $\mathrm{x}$ & $\mathrm{x}$ & \\
\hline Thrasya glaziovii A. G. Burm. & Erva & 8 & $\mathrm{x}$ & & & & & $\mathrm{x}$ \\
\hline Thrasya petrosa (Trin.) Chase & Erva & 50 & $\mathrm{x}$ & $\mathrm{x}$ & & $\mathrm{x}$ & $\mathrm{x}$ & $\mathrm{x}$ \\
\hline Trachypogon spicatus (L. f.) Kuntze * & Erva & 96 & $\mathrm{x}$ & $\mathrm{x}$ & $\mathrm{x}$ & $\mathrm{x}$ & $\mathrm{x}$ & $\mathrm{x}$ \\
\hline \multicolumn{9}{|l|}{ POLYGALACEAE } \\
\hline Monnina exalata A. W. Benn. & Erva & 50 & & $\mathrm{x}$ & $\mathrm{x}$ & $\mathrm{x}$ & $\mathrm{x}$ & $\mathrm{x}$ \\
\hline Polygala cuspidata DC. & Erva & 8 & $\mathrm{x}$ & $\mathrm{x}$ & & & & \\
\hline Polygala galioides Poir. & Erva & 45 & $\mathrm{x}$ & $\mathrm{x}$ & $\mathrm{x}$ & $\mathrm{x}$ & $\mathrm{x}$ & $\mathrm{x}$ \\
\hline Polygala hebeclada DC. & Erva & 4 & & & & $\mathrm{x}$ & & \\
\hline Polygala hirsuta A. St.-Hil. \& Moq. & Erva & 4 & & & & & & $\mathrm{x}$ \\
\hline Polygala minima Pohl ex A. W. Benn. & Erva & 8 & & & $\mathrm{x}$ & $\mathrm{x}$ & & \\
\hline Polygala violacea Aubl. & Erva & 41 & $\mathrm{x}$ & $\mathrm{x}$ & $\mathrm{x}$ & $\mathrm{x}$ & $\mathrm{x}$ & $\mathrm{x}$ \\
\hline \multicolumn{9}{|l|}{ PROTEACEAE } \\
\hline Roupala montana Aubl. & Árvore & 8 & $\mathrm{x}$ & & & $\mathrm{x}$ & & \\
\hline \multicolumn{9}{|l|}{ RHAMAMNACEAE } \\
\hline Crumenaria chorethroides Mart. ex Reissek & Erva & 100 & $\mathrm{x}$ & $\mathrm{x}$ & $\mathrm{x}$ & $\mathrm{x}$ & $\mathrm{x}$ & $\mathrm{x}$ \\
\hline \multicolumn{9}{|l|}{ RUBIACEAE } \\
\hline Mitracarpus parvulus K. Schum. & Erva & 4 & & & & & $\mathrm{x}$ & \\
\hline Palicourea officinalis Mart. & Subarbusto & 100 & $\mathrm{x}$ & $\mathrm{x}$ & $\mathrm{x}$ & $\mathrm{x}$ & $\mathrm{x}$ & $\mathrm{x}$ \\
\hline Palicourea rigida Kunth & Arbusto, árvore & 12 & & $\mathrm{x}$ & & $\mathrm{x}$ & $\mathrm{x}$ & \\
\hline Richardia brasiliensis Gomes & Erva & 16 & $\mathrm{x}$ & & & $\mathrm{x}$ & $\mathrm{x}$ & \\
\hline Sabicea brasiliensis Wernham & Subarbusto, arbusto & 12 & & $\mathrm{x}$ & $\mathrm{x}$ & & $\mathrm{x}$ & \\
\hline Sipania hispida Benth. ex Wernham & Erva & 16 & & & & $\mathrm{x}$ & & $\mathrm{x}$ \\
\hline Spermacoce capitata Ruiz \& Pavon & Erva & 62 & $\mathrm{x}$ & $\mathrm{x}$ & $\mathrm{x}$ & $\mathrm{x}$ & $\mathrm{x}$ & $\mathrm{x}$ \\
\hline Spermacoce poaya A. St.-Hil. & Erva & 16 & $\mathrm{x}$ & & $\mathrm{x}$ & & & $\mathrm{x}$ \\
\hline Spermacoce schumanniana (Taub. ex Ule) Govaerts & Subarbusto & 54 & $\mathrm{x}$ & & $\mathrm{x}$ & $\mathrm{x}$ & $\mathrm{x}$ & $\mathrm{x}$ \\
\hline Spermacoce tenella Kunth & Subarbusto & 8 & $\mathrm{x}$ & & & & & $\mathrm{x}$ \\
\hline Spermacoce warmingii (K. Schum. ex Mart.) Kunth & Erva & 16 & $\mathrm{x}$ & & $\mathrm{x}$ & & & $\mathrm{x}$ \\
\hline \multicolumn{9}{|l|}{ RUTACEAE } \\
\hline Zanthoxylum rhoifolium Lam. & Árvore & 64 & $\mathrm{x}$ & $\mathrm{x}$ & $\mathrm{x}$ & $\mathrm{x}$ & $\mathrm{x}$ & $\mathrm{x}$ \\
\hline \multicolumn{9}{|l|}{ SALICACEAE } \\
\hline Casearia altiplanensis Sleumer & Arbusto & 41 & $\mathrm{x}$ & $\mathrm{x}$ & $\mathrm{x}$ & $\mathrm{x}$ & $\mathrm{x}$ & $\mathrm{x}$ \\
\hline Casearia sylvestris $\mathrm{Sw}$. & Arbusto & 87 & $\mathrm{x}$ & $\mathrm{x}$ & $\mathrm{x}$ & $\mathrm{x}$ & $\mathrm{x}$ & $\mathrm{x}$ \\
\hline \multicolumn{9}{|l|}{ SAPINDACEAE } \\
\hline Matayba guianenesis Aubl. & Árvore & 8 & $\mathrm{x}$ & & $\mathrm{x}$ & & & \\
\hline Serjania caracasana (Jacq.) Willd. & Trepadeira & 4 & & $\mathrm{x}$ & & & & \\
\hline Serjania erecta Radlk. & Trepadeira & 29 & $\mathrm{x}$ & $\mathrm{x}$ & $\mathrm{x}$ & & $\mathrm{x}$ & $\mathrm{x}$ \\
\hline \multicolumn{9}{|l|}{ SCHIZEACEAE } \\
\hline Anemia pastinacaria Moritz ex Prantl. & Erva & 29 & $\mathrm{x}$ & $\mathrm{x}$ & $\mathrm{x}$ & & & $\mathrm{x}$ \\
\hline \multicolumn{9}{|l|}{ SIMAROUBACEAE } \\
\hline \multicolumn{8}{|l|}{ SMILACACEAE } & \\
\hline Smilax goyazana A. DC. & Subarbusto & 91 & $\mathrm{x}$ & $\mathrm{x}$ & $\mathrm{x}$ & $\mathrm{x}$ & $\mathrm{x}$ & $\mathrm{x}$ \\
\hline \multicolumn{9}{|l|}{ SOLANACEAE } \\
\hline Schwenckia americana D. Royen ex L. & Erva & 41 & $\mathrm{x}$ & $\mathrm{x}$ & $\mathrm{x}$ & $\mathrm{x}$ & $\mathrm{x}$ & $\mathrm{x}$ \\
\hline Solanum aff. lycocarpum A. St.-Hil. & Arbusto, árvore & 33 & $\mathrm{x}$ & $\mathrm{x}$ & $\mathrm{x}$ & & $\mathrm{x}$ & \\
\hline Solanum subumbellatum Vell. & Subarbusto, arbusto & 58 & $\mathrm{x}$ & $\mathrm{x}$ & $\mathrm{x}$ & $\mathrm{x}$ & $\mathrm{x}$ & $\mathrm{x}$ \\
\hline Solanum sp. (CRM 220) & Erva & 33 & $\mathrm{x}$ & $\mathrm{x}$ & $\mathrm{x}$ & $\mathrm{x}$ & $\mathrm{x}$ & $\mathrm{x}$ \\
\hline \multicolumn{9}{|l|}{ TURNERACEAE } \\
\hline $\begin{array}{l}\text { Piriqueta sidifolia (A. St.-Hil., A. Juss. \& Cambess.) } \\
\text { Urb. }\end{array}$ & Erva & 29 & & $\mathrm{x}$ & & $\mathrm{x}$ & $\mathrm{x}$ & $\mathrm{x}$ \\
\hline Turnera lamiifolia Cambess. & Erva & 75 & $\mathrm{x}$ & $\mathrm{x}$ & $\mathrm{x}$ & $\mathrm{x}$ & $\mathrm{x}$ & $\mathrm{x}$ \\
\hline Turnera nana Cambess. & Erva & 50 & & & $\mathrm{x}$ & $\mathrm{x}$ & $\mathrm{x}$ & $\mathrm{x}$ \\
\hline
\end{tabular}


continuação

\begin{tabular}{|c|c|c|c|c|c|c|c|c|}
\hline Família/Espécie & Hábito & Freq $^{1}$ & $\mathrm{CM}$ & $\mathrm{CS}$ & FM & FS & MIM & MIS \\
\hline \multicolumn{9}{|l|}{ URTICACEAE } \\
\hline Cecropia pachystachya Trécul & Árvore & 4 & & & & $\mathrm{x}$ & & \\
\hline \multicolumn{9}{|l|}{ VERBENACEAE } \\
\hline Lantana camara L. * & Arbusto & 4 & & & & & & $\mathrm{x}$ \\
\hline Lantana hypoleuca Briq. & Arbusto & 87 & $\mathrm{x}$ & $\mathrm{x}$ & $\mathrm{x}$ & $\mathrm{x}$ & $\mathrm{x}$ & $\mathrm{x}$ \\
\hline Lippia elegans Cham. & Arbusto & 4 & & & & & & $\mathrm{x}$ \\
\hline Lippia grandiflora Mart. \& Schauer & Subarbusto & 12 & & & $\mathrm{x}$ & & $\mathrm{x}$ & \\
\hline Lippia lacunosa Mart. \& Schauer & Arbusto & 91 & $\mathrm{x}$ & $\mathrm{x}$ & $\mathrm{x}$ & $\mathrm{x}$ & $\mathrm{x}$ & $\mathrm{x}$ \\
\hline Lippia martiana Schauer & Arbusto & 37 & & $\mathrm{x}$ & & $\mathrm{x}$ & $\mathrm{x}$ & $\mathrm{x}$ \\
\hline Lippia pumila Cham. & Arbusto & 12 & & $\mathrm{x}$ & & $\mathrm{x}$ & & $\mathrm{x}$ \\
\hline Lippia sericea Cham. & Subarbusto & 33 & $\mathrm{x}$ & $\mathrm{x}$ & $\mathrm{x}$ & $\mathrm{x}$ & $\mathrm{x}$ & $\mathrm{x}$ \\
\hline Stachytarpheta cayennensis (L. C. Rich.) Vahl & Erva, subarbusto & 16 & $\mathrm{x}$ & & $\mathrm{x}$ & $\mathrm{x}$ & & $\mathrm{x}$ \\
\hline \multicolumn{9}{|l|}{ VIOLACEAE } \\
\hline Hybanthus lanatum (A. St.-Hil.) Baill. & Erva & 33 & $\mathrm{x}$ & & $\mathrm{x}$ & & $\mathrm{x}$ & $\mathrm{x}$ \\
\hline \multicolumn{9}{|l|}{ VOCHYSIACEAE } \\
\hline Qualea cordata (Mart.) Spreng. & Árvore & 12 & $\mathrm{x}$ & & & & $\mathrm{x}$ & $\mathrm{x}$ \\
\hline Qualea grandiflora Mart. & Árvore & 33 & $\mathrm{x}$ & $\mathrm{x}$ & $\mathrm{x}$ & $\mathrm{x}$ & & $\mathrm{x}$ \\
\hline Qualea multiflora Mart. & Árvore & 4 & $\mathrm{x}$ & & & & & \\
\hline Qualea parviflora Mart. & Árvore & 54 & $\mathrm{x}$ & $\mathrm{x}$ & $\mathrm{x}$ & $\mathrm{x}$ & $\mathrm{x}$ & $\mathrm{x}$ \\
\hline Vochysia tucanorum (Spreng.) Mart. & Árvore & 4 & & & & $\mathrm{x}$ & & \\
\hline
\end{tabular}

1. Porcentagem das parcelas em que a espécie ocorre / 1. Percent of parcel where the species occurs.

* Espécies exóticas indicadas em Mendonça et al. (2008) / * Exoctic species according to Mendonça et al. (2008)

apesar da presença significativa do capim-gordura na área, a riqueza em espécies manteve-se alta.

Das espécies registradas, $14(3,48 \%)$ foram encontradas em todas as parcelas estudadas (indicadas por freq $=100$ na tabela 1$)$, enquanto $60(14,92 \%)$ foram encontradas em apenas uma parcela (freq $=4$ na tabela 1). Trinta e sete espécies $(9,20 \%)$ são consideradas exóticas para o Bioma Cerrado segundo Mendonça et al. (2008) (indicadas por asterisco na tabela 1). O componente subarbustivo-herbáceo (que inclui ervas, subarbustos, hemiparasitas, trepadeiras e uma palmeira acaule) apresentou o maior número de espécies (cerca de 67\%), comparado com as espécies de hábito arbustivoarbóreo, grupo que também incluiu palmeiras arbustivas, com cerca de $33 \%$. Essa característica da distribuição de riqueza mais elevada no componente subarbustivoherbáceo deve-se principalmente a contribuição das espécies do estrato rasteiro, em plantas pertencentes às famílias Asteraceae, Poaceae e Leguminosae.

Estes resultados evidenciam a considerável riqueza florística na área experimental estudada, apesar da presença de capim-gordura, particularmente quando se compara com as cerca de 1.230 espécies listadas até o presente para o PNB (Funatura/Ibama 1998, Martins et al. 2007). Neste caso, a área amostral conteria $32,68 \%$ de todas as espécies dessa unidade de conservação. Assim, o levantamento realizado neste trabalho reforça a constatação de Funatura/Ibama (1998) e de Proença et al. (2001), que indicaram que a flora fanerogâmica do Parque é apenas parcialmente conhecida.

Nos tratamentos fogo/maio, fogo/setembro, manejo integrado/maio e manejo integrado/setembro, a queima controlada exerceu um papel importante como instrumento de poda, ou abertura de área. Registrese que, nesses tratamentos, para muitas espécies houve estímulo à produção de flores e frutos, fato não constatado em vários indivíduos das mesmas espécies ocorrendo nos tratamentos controle. Entre as espécies que floresceram pós-fogo cita-se: Baccharis humilis e Chrysolaena herbacea (Asteraceae); Acalypha claussenii (Euphorbiaceae); Marsypianthes montana (Lamiaceae); Clitoria guianensis (Leguminosae); Tetrapterys ambigua (Malpighiaceae); Waltheria communis (Malvaceae); Anthaenantia lanata, Elionurus muticus e Paspalum erianthum (Poaceae); Crumenaria chorethroides (Rhamnaceae). Essa informação corroborou Coutinho (1980) e outros autores como César (1980), que registraram comportamento semelhante aos observados neste estudo em área do DF próxima ao PNB. A floração dessas espécies poderia resultar na produção de sementes, as quais facilitariam a permanência das espécies na área, bem como possibilitariam a colonização de outras áreas.

$\mathrm{Na}$ área experimental, a biomassa do estrato rasteiro encontrada foi de $8 \mathrm{t} \mathrm{ha}^{-1}$ em dezembro de 2002 , 
sendo que o capim-gordura representava cerca de $62 \%$ desse total. Onde o capim-gordura formava "stands" monoespecíficos, observou-se que a biomassa aérea total variou entre 12,1 a $21,4 \mathrm{t} \mathrm{ha}^{-1}$. Estes resultados sinalizam que essa gramínea apresenta um grande potencial para modificar a biomassa do estrato rasteiro das áreas invadidas, pois, de acordo com Miranda et al. (2004), dependendo da forma fisionômica considerada do Cerrado e do período de proteção contra o fogo, a biomassa do estrato rasteiro pode variar entre 6,9 a $10 \mathrm{t} \mathrm{ha}^{-1}$. Assim sendo, o comportamento apresentado pelo capim-gordura reforça que esta gramínea está bem adaptada ao clima da região Centro-Oeste do Brasil, como também está adaptada aos solos do bioma, que geralmente apresentam baixa soma de bases trocáveis ( $\mathrm{K}, \mathrm{Ca}, \mathrm{Mg}$ ), acidez alta, resultando em altos índices de saturação por alumínio (Batmanian \& Haridasan 1985).

A dinâmica da recuperação da vegetação do estrato rasteiro nas áreas dos tratamentos avaliados mostrou que, no segundo ano após a implantação do experimento, a biomassa total registrada nos tratamentos fogo/maio, fogo/setembro, manejo integrado/maio e manejo integrado/setembro alcançou 5,6 $\mathrm{t} \mathrm{ha}^{-1}$, 4,5 $\mathrm{t} \mathrm{ha}^{-1}, 3,8 \mathrm{tha}^{-1}$ e 3,5 t ha-1 ${ }^{-1}$, respectivamente. Estes valores representam uma recuperação da biomassa total de $63 \%, 56 \%, 45 \%$ e $52 \%$, respectivamente, e diferem significativamente dos valores encontrados antes da intervenção na área experimental $(t=5,22$ e $P<0,0001 ; t=5,22$ e $P<0,0001 ; t=7,30$ e $P<0,0001 ; t=5,10$ e $P<0,0001)$ (figura 3). Por outro lado, nos tratamentos controle/maio e controle/setembro, a biomassa total apresentou uma redução natural de $13 \%$ e $17 \%$, respectivamente, sendo que apenas no segundo tratamento houve diferença significativa do valor encontrado quando comparado com o registrado no início do experimento de campo $(t=1,67$ e $P=0,1130 ; t=2,18$ e $P=0,0428$ ) (figura 3).

No mesmo período, a biomassa do capim-gordura nos tratamentos fogo/maio, fogo/setembro, manejo integrado/ maio e manejo integrado/setembro alcançou $1,6 \mathrm{t} \mathrm{ha}^{-1}$, $0,9 \mathrm{t} \mathrm{ha}^{-1}, 0,021 \mathrm{t} \mathrm{ha}^{-1}$ e $0,020 \mathrm{tha}^{-1}$, respectivamente. Estes valores representam uma recuperação da biomassa do capim-gordura de 34\%, 21\%, 0,36\% e $0,41 \%$, respectivamente, e diferem significativamente dos valores encontrados antes da intervenção na área experimental $(t=5,14$ e $P<0,0001 ; t=5,94 \mathrm{e}$ $P<0,0001 ; t=9,55$ e $P<0,0001 ; t=7,72$ e $P<0,0001)$ (figura 3). Nos tratamentos controle/maio e controle/ setembro a biomassa do capim-gordura apresentou uma queda natural de $54 \%$ e $48 \%$, respectivamente. Esta diferença também é significativa $(t=4,23$ e $P<0,0005$; $t=3,25$ e $P=0,0045)$ quando se compara com os valores encontrados no início do experimento de campo (figura 3).
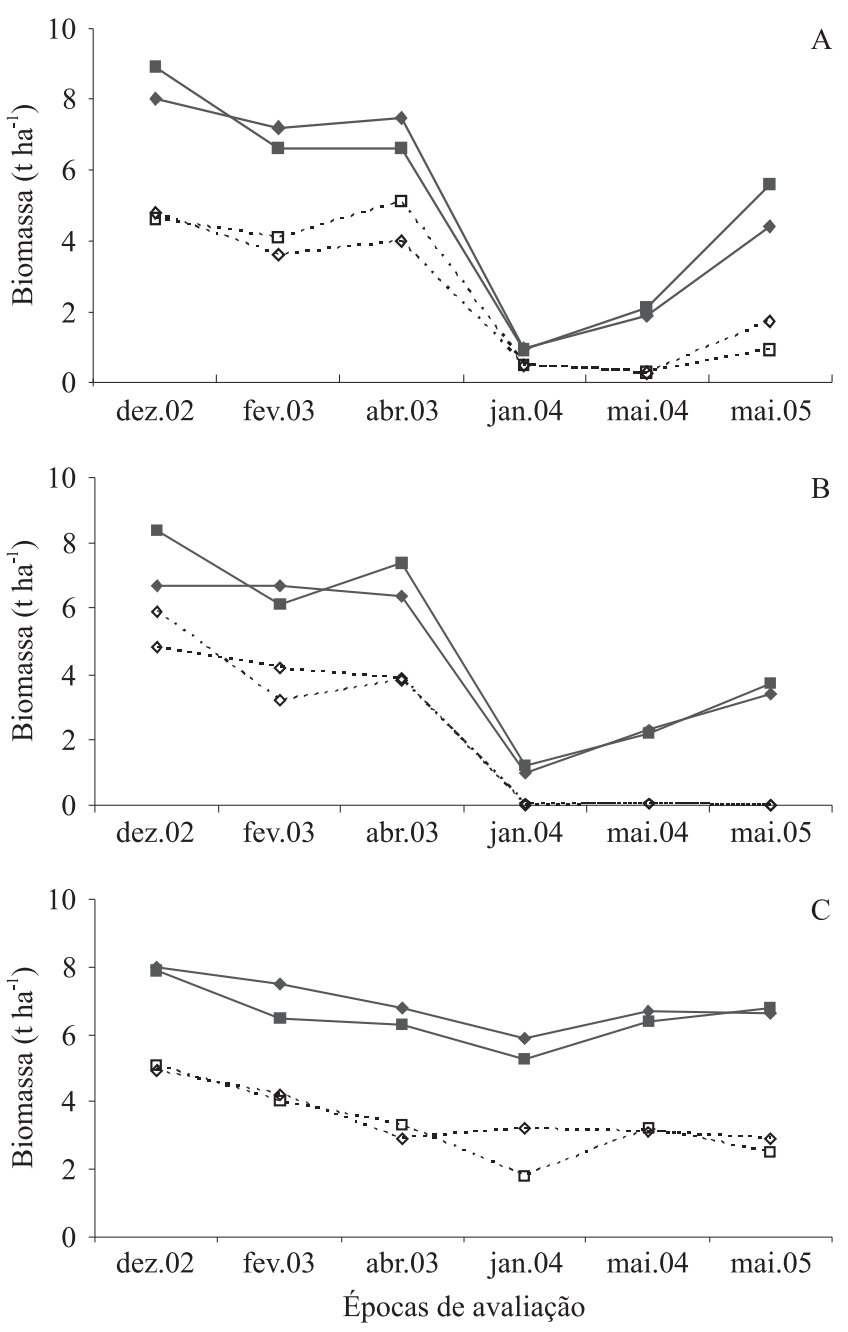

Figura 3. Recuperação da biomassa total e do capim-gordura nos tratamentos. A. Fogo/maio (FM) e Fogo/setembro (FS). B. Manejo integrado/maio (MIM) e Manejo integrado/setembro (MIS). C. Controle/maio (CM) e Controle/setembro (CS), após 23 e 19 meses, respectivamente, no Parque Nacional de Brasília, Distrito Federal. Maio, biomassa total ( $\square$ ); maio, biomassa capim-gordura $(\square)$; setembro, biomassa total $(\diamond) \mathrm{e}$ setembro, biomassa capim-gordura $(\diamond)$.

Figure 3. Recovery of total biomass and of molasses grass biomass in the treatments. A. Fire/May (FM) and Fire/ September (FS). B. Treatments Integrated Management/ May (MIM) and Integrated Management/September (MIS). C. Control/May (CM) and Control/September (CS), after 23 and 19 months, respectively, in the National Park of Brasilia, Federal District. May total biomass (ם), May molasses grass biomass $(\square)$, September total biomass ( $\bullet$ ), September molasses grass biomass $(\diamond)$. 
No que tange à comparação entre a biomassa total em 2005, os resultados mostraram que houve diferença significativa entre ambos os tratamentos de manejo integrado e os controles $\left(F_{2,2}=23,05\right.$ e $\left.P<0,0001\right)$ (tabela 2). Por sua vez, a biomassa do capim-gordura nos tratamentos controle e fogo diferem significativamente da registrada no manejo integrado e são semelhantes entre si $\left(F_{2,2}=9,08\right.$ e $\left.P=0,0026\right)$ (tabela 2).

Tabela 2. Biomassa total e do capim-gordura (t/ha) nos tratamentos Controle/maio (CM), Controle/setembro (CS), Fogo/maio (FM), Fogo/setembro (FS), Manejo integrado/ maio (MIM) e Manejo integrado/setembro (MIS), após 23 e 19 meses, respectivamente, no Parque Nacional de Brasília, Distrito Federal. Médias seguidas da mesma letra em cada coluna não diferem pelo teste de Tukey a $5 \%$ de probabilidade.

Table 2. Total biomass and molasses grass biomass $\left(\mathrm{t} \mathrm{ha}^{-1}\right)$ in the treatments Control/May (CM), Control/September (CS), Fire/May (FM), Fire/September(FS), Integrated Management/ May (MIM) and Integrated Management/September MIS) after 23 and 19 months, respectively, in the National Park of Brasilia, Federal District. Means followed by the same letter in each column are not statistically different, using a Tukey test with $P=0.05$.

\begin{tabular}{llcc}
\hline \multirow{2}{*}{ Tratamentos } & & \multicolumn{2}{c}{ Biomassa $\left(\mathrm{t} \mathrm{ha}^{-1}\right)$} \\
\cline { 3 - 4 } \cline { 3 - 4 } & \multicolumn{1}{c}{ total } & capim-gordura \\
\cline { 3 - 4 } CM & & $6,8( \pm 1,2) \mathrm{a}$ & $2,5( \pm 1,5) \mathrm{a}$ \\
$\mathrm{CS}$ & & $6,6( \pm 0,9) \mathrm{a}$ & $2,9( \pm 2,5) \mathrm{a}$ \\
FM & & $5,6( \pm 1,1) \mathrm{ab}$ & $1,6( \pm 1,2) \mathrm{a}$ \\
FS & & $4,4( \pm 1,1)) \mathrm{ab}$ & $0,9( \pm 0,4) \mathrm{a}$ \\
MIM & & $3,7( \pm 0,7) \mathrm{b}$ & $0,022( \pm 0,017) \mathrm{b}$ \\
MIS & & $3,4( \pm 0,6) \mathrm{b}$ & $0,020( \pm 0,018) \mathrm{b}$ \\
\hline
\end{tabular}

A dinâmica da biomassa registrada nas áreas invadidas pelo capim-gordura está em conformidade com dados de Brooks \& Pyke (2001) para regiões áridas norte-americanas, que indicaram que as invasões biológicas podem mudar a biomassa das áreas invadidas. Resultado semelhante também foi relatado por Rossiter et al. (2003), que indicaram a gramínea africana Andropogon gayanus como sendo responsável pelo aumento significativo da biomassa do estrato rasteiro em áreas de savanas australianas.

Os resultados obtidos mostram que, na área experimental, em decorrência do processo de estabelecimento e colonização do capim-gordura, a biomassa do estrato rasteiro sofreu uma mudança na sua composição (62\% capim-gordura e $38 \%$ vegetação nativa). Contudo, o número de espécies nativas nesse ambiente é expressivo, o que revela que a vegetação ainda é capaz de conviver com o capim-gordura nos atuais níveis de infestação alcançados por essa gramínea.

Investigações sobre os limites do índice de cobertura, acima do qual a presença do capim-gordura afeta a riqueza de espécies nativas da área invadida, são inexistentes. $\mathrm{Na}$ Austrália, Gooden et al. (2009) mostraram que em áreas invadidas por Lantana camara a riqueza de espécies nativas manteve-se estável, onde a sua cobertura foi inferior a $75 \%$. Contudo, houve um rápido declínio no número das espécies nativas nas áreas onde a cobertura dessa invasora excedeu os $75 \%$. Por outro lado, Turner et al. (2008) registraram que nas áreas onde a cobertura da espécie invasora Asparagus asparagoides (L.) Druce variou entre $40,3 \%$ e $61,7 \%$, o número de espécies nativas foi $52 \%$ menor quando comparado com áreas onde essa espécie estava ausente.

Dois anos após o início do presente experimento de campo, constatou-se que a realização de uma queimada controlada não foi suficiente para controlar o capim-gordura, pois a sua biomassa encontrava-se em recuperação. Essa constatação corrobora Filgueiras (1990) e Martins et al. (2006), que citaram que o fogo não elimina o capim-gordura.

$\mathrm{Na}$ área submetida ao manejo integrado a biomassa do capim-gordura alcançou $0,056 \%$ do valor registrado em dezembro de 2002. Neste caso, em decorrência das sementes do capim-gordura permanecerem viáveis no solo por cerca de dois anos (Martins 2006), recomenda-se que as áreas submetidas a esse tratamento sejam monitoradas por pelo menos três anos. É provável que novas intervenções devam ser realizadas neste período para prevenir a reinfestação dessa gramínea nesses ambientes.

Nas condições estudadas constatou-se que a colonização do capim-gordura alterou significativamente a biomassa do estrato rasteiro. Nas áreas onde a gramínea forma densas populações, sua biomassa alcançou o dobro da que é citada para a vegetação do estrato rasteiro da região.

Apesar do nível de infestação do capim gordura, o número de espécies nativas registrado foi expressivo, o que mostra que a vegetação nativa apresenta resistência ao processo de invasão do capim gordura, pelo menos em áreas onde esta gramínea não ultrapassa em torno de $60 \%$ do total da biomassa presente. Se isto se revelará em resiliência é algo ainda a ser investigado.

A biomassa do capim gordura no tratamento fogo encontra-se em recuperação, o que sinaliza que a realização de uma queimada não é suficiente para controlar a espécie. Por outro lado, a biomassa dessa 
gramínea no manejo integrado foi reduzida em mais de $99,9 \%$, o que se configura como uma promissora estratégia de manejo que favorece a expansão da vegetação nativa do estrato rasteiro no Cerrado.

Por ser uma espécie que apresenta grande potencial para colonizar áreas naturais e antropizadas, ações de manejo para o controle do capim-gordura deverão ser implementadas pelos gestores de áreas protegidas. Nas unidades de conservação onde não se registrou a sua presença, recomenda-se a implantação de um programa de monitoramento constante para evitar seu estabelecimento e expansão. Neste caso, os focos iniciais de sua ocorrência deverão ser imediatamente erradicados. Por outro lado, nas unidades de conservação onde o capim-gordura já está estabelecido, intervenções de manejo integrado para seu controle deverão ser conduzidas, cujo objetivo é favorecer a expansão da vegetação nativa das áreas invadidas por essa gramínea.

Agradecimentos - Ao Instituto Chico de Mendes de Conservação da Biodiversidade (ICMBio) e ao Parque Nacional de Brasília, pela autorização para realizar o trabalho. A WWF/Brasil (Código BRZ NT 614/2002) e The Nature Conservancy Brasil (Doação № 020/03) pelo apoio financeiro, e Monsanto do Brasil pelo apoio técnico. Ao colega Sérgio Eustáquio de Noronha, pela confecção do mapa.

\section{Referências bibliográficas}

APG II. 2003. An update of the Angiosperm Phylogeny Group classification for the orders and families of flowering plants: APG II. Botanical Journal of the Linnean Society 141:399-436.

BATMANIAN, B.G. \& HARIDASAN, M. 1985. Primary production and accumulation of nutrients by the ground layer community of cerrado vegetation of central Brazil. Plant and Soil 88:437-440.

BROOKS, M.L. \& PYKE, D.A. 2001. Invasive plants and fire in the desert of North America. In Proceedings of the invasive species workshop: the role of fire in the control and spread of invasive species. (K.E.M. Galley \& T.P. Wilson, eds.). Fire Conference 2000: the First National Congress on Fire Ecology, Prevention, and Management Miscellaneous Publication № 11, Tall Timbers Research Station, Tallahassee, p.1-14.

CÉSAR, H. 1980. Efeito da queimada e corte sobre a vegetação de um campo sujo na Fazenda Água Limpa, Brasília / DF. Dissertação de mestrado, Universidade de Brasília, Brasília.

COELHO, H.A. 2002. Histórico de regime de fogo do Parque Nacional de Brasília. Monografia de final de curso, Departamento de Engenharia Florestal, Universidade de Brasília, Brasília.
COUTINHO, L.M. 1980. As queimadas e seu papel ecológico. Brasil Florestal 44:7-23.

D’ANTONIO, C.M. \& VITOUSEK, P.M. 1992. Biological invasions by exotic grasses, the grass/fire cycle, and global change. Annual Review of Ecology and Systematics 23:63-87.

FILGUEIRAS, T.S. 1990. Africanas no Brasil: gramíneas introduzidas da África. Cadernos de Geociências 5:57-63.

FILGUEIRAS, T.S., NOGUEIRA, P.E, BRACHADO, A.L. \& GUALA, G.F. 1994. Caminhamento - um método expedito para levantamentos florísticos qualitativos. Cadernos de Geociências 12:39-43.

FUNATURA/IBAMA. 1998. Plano de Manejo do Parque Nacional de Brasília/Revisão. Fundação Pró-Natureza e Instituto Brasileiro do Meio Ambiente e dos Recursos Naturais Renováveis, Brasília. v.1.

GOODEN, B., FRENCH, V. \& TURNER, P.J. 2009. Invasion and management of wood plant, Lantana camara L., alters vegetation diversity within wet sclerophyll forest in southeastern Australia. Forest Ecology and Management 257:960-967.

ISHARA, K.L., DÉSTRO, G.F.G., MAIMONI-RODELLA, R.C.S. \& YANAGIZAWA, Y.A.N.P. 2008. Composição florística de remanescente de cerrado sensu stricto em Botucatu, SP. Revista Brasileira de Botânica 31:575-586.

MARTINS, C.R., HAY, J.D.V., CARMONA, R., LEITE, R.R., SCALÉA, M., VIVALDI, L.J. \& PROENÇA, C.E.B. 2004. Monitoramento e controle da gramínea invasora Melinis minutiflora (capim-gordura) no Parque Nacional de Brasília, Distrito Federal. In Anais do IV Congresso Brasileiro de Unidades de Conservação. Seminário 2. Fundação O Boticário de Proteção à Natureza, Rede Nacional Pró Unidade de Conservação. Curitiba, p.85-96.

MARTINS, C.R. 2006. Caracterização e manejo da gramínea Melinis minutiflora P. Beauv. (capim-gordura): uma espécie invasora do cerrado. Tese de doutorado, Universidade de Brasília, Brasília.

MARTINS, C.R., HAY, J.D., VALLS, J.F.M., LEITE, L.L. \& HENRIQUES, R.P.B. 2007. Levantamento das gramíneas exóticas do Parque Nacional de Brasília, Distrito Federal, Brasil. Natureza \& Conservação 5:23-30.

MCNEELY, J.A., MOONEY, H.A., NEVILLE, L.E., SCHEI, P. \& WAAGE, J.K. (EDS.). 2001. A global strategy on invasive alien species. IUCN Gland, Switzerland, and Cambridge.

MENDONÇA, R.C.,FELFILI, J.M., WALTER, B.M.T., SILVAJÚNIOR, M.C., REZENDE, A.V., FILGUEIRAS, T.S., NOGUEIRA, P.E. \& FAGG, C.W. 2008. Flora vascular do bioma Cerrado: um checklist com 12.356 espécies. In Cerrado: ecologia e flora. (S.M. Sano, S.P. Almeida \& J.F. Ribeiro, eds.). Embrapa Cerrados/Embrapa Informação Tecnológica, Brasília v.2. p.421-1279.

MIDITIERI, J. 1983. Manual de gramíneas e leguminosas para pastos tropicais. Nobel / Ed. da Universidade de São Paulo, São Paulo. 
MIRANDA, H.S., SATO, M.N., ANDRADE, S.M., HARIDASAN, M. \& MORAIS, H.C. 2004. Queimadas de Cerrado: caracterização e impactos. In Cerrado: ecologia e caracterização. (L.M.S. Aguiar, \& A.J.A. Camargo, eds.). Brasília, Embrapa Cerrados, p.69-123.

MUNHOZ, C.B.R. \& FELFILI, J.M. 2006. Fitossociologia do estrato herbáceo-subarbustivo em uma área de campo sujo no Distrito Federal, Brasil. Acta Botanica Brasilica 20:671-685.

MUNHOZ, C.B.R. \& FELFILI, J.M. 2008. Fitossociologia do estrato herbáceo-subarbustivo em um campo limpoúmido no Brasil Central. Acta Botanica Brasilica 22:905-913.

PINTO, J.R.R., LENZA, E. \& PINTO, A.S. 2009. Composição florística e estrutura da vegetação arbustivo-arbórea em um cerrado rupestre, Cocalzinho de Goiás, Goiás. Revista Brasileira de Botânica 32:1-10.

PIVELLO,V.R.,CARVALHO,V.M.C.,LOPES,P.F.,PECCININI, A.A. \& ROSO, S. 1999a. Abundance and distribution of native and alien grasses in a "cerrado" (Brazilian savanna) biological reserve. Biotropica 31:71-82.

PIVELLO, V.R., SHIDA, C.N. \& MEIRELLES, S.T. $1999 \mathrm{~b}$. Alien grasses in Brazilian savannas: a threat to the biodiversity. Biodiversity and Conservation 8:1281-1294.

PROENÇA, C.E.B., MUNHOZ, C.B.R., JORGE, C.L. \& NOBREGA, M.G.G. 2001. Listagem e nível de proteção das espécies de fanerógamas do Distrito Federal. In Flora do Distrito Federal, Brasil. (T.B. Cavalcanti \& A.E. Ramos, org.). Embrapa Recursos Genéticos e Biotecnologia, Brasília, v.1, p.89-359.
RIBEIRO, J.F. \& WALTER, B.M.T. 2008. As principais fitofisionomias do bioma Cerrado. In Cerrado: ecologia e flora. (S.M. Sano, S.P. Almeida \& J.F. Ribeiro, eds.). Embrapa Cerrados/Embrapa Informação Tecnológica, Brasília, v.1, p.151-212.

ROSSITER, N.A., SETTERFIELD, S.A., DOUGLAS, M.M. \& HUTLEY, L.B. 2003. Testing the grass-fire cycle: alien grass invasion in the tropical savannas of northern Australia. Diversity and Distributions 9: 169-176.

SAN-JOSÉ, J.J. \& FARIÑAS, M.R. 1991. Temporal changes in the structure of Trachypogon savanna protected for 25 years. Acta Ecologica 12:237-287.

SAS INSTITUTE. 2003. PROC MIXED User's Guide. Versão 9.1.SAS, Cary.

SOUZA, V.C. \& LORENZI, H. 2008. Botânica sistemática: guia ilustrado para identificação das famílias de fanerógamas nativas e exóticas no Brasil, baseado em APG II. 2a ed., Instituto Plantarum, Nova Odessa.

TANNUS, J.L.S. \& ASSIS, M.A. 2004. Composição de espécies vasculares de campo sujo e campo úmido em área de cerrado, Itirapina / SP, Brasil. Revista Brasileira de Botânica 27:489-506.

TURNER, P.J., SCOTT, J.K. \& SPAFFORD, H. 2008. The ecological barriers to the recovery of bridal creeper (Asparagus asparagoides (L.) Druce) infested sites: impacts on vegetation and the potential increase in other exotic species. Austral Ecology 33: 713-722. 